\title{
Frieze patterns for punctured discs
}

\author{
Karin Baur • Bethany R. Marsh
}

Received: 11 December 2007 / Accepted: 29 October 2008 / Published online: 20 December 2008 (C) Springer Science+Business Media, LLC 2008

\begin{abstract}
We construct frieze patterns of type $D_{N}$ with entries which are numbers of matchings between vertices and triangles of corresponding triangulations of a punctured disc. For triangulations corresponding to orientations of the Dynkin diagram of type $D_{N}$, we show that the numbers in the pattern can be interpreted as specialisations of cluster variables in the corresponding Fomin-Zelevinsky cluster algebra. This is generalised to arbitrary triangulations in an appendix by Hugh Thomas.
\end{abstract}

Keywords Cluster algebra - Frieze pattern - Ptolemy rule · Exchange relation · Matching $\cdot$ Riemann surface $\cdot$ Disc $\cdot$ Triangulation

\section{Introduction}

Frieze patterns were introduced by Conway and Coxeter in [8, 9]. Such a pattern consists of a finite number of rows arranged in an array so that the numbers in the $k$ th row sit between the numbers in the rows on either side. The first and last rows consist $b$

of ones and for every diamond of the form $a \quad d$ the relation $a d-b c=1$ must be $c$

The authors were supported by an EPSRC grant, number GR/S35387/01.

With an Appendix by Hugh Thomas.

K. Baur

Department of Mathematics, ETH Zürich, Rämistrasse 101, 8092 Zürich, Switzerland

e-mail: baur@math.ethz.ch

B.R. Marsh $\varangle)$

Department of Pure Mathematics, University of Leeds, Leeds LS2 9JT, UK

e-mail: B.R.Marsh@leeds.ac.uk 
Fig. 1 A Conway-Coxeter frieze pattern of order 6

$\begin{array}{cccccccccccccccc} & 1 & & 1 & & 1 & & 1 & & 1 & & 1 & & 1 & \\ & & 1 & & 4 & & 1 & & 2 & & 2 & & 2 & & \\ \cdots & 1 & & 3 & & 3 & & 1 & & 3 & & 3 & & 1 & \cdots \\ & & 2 & & 2 & & 2 & & 1 & & 4 & & 1 & & \\ & 1 & & 1 & & 1 & & 1 & & 1 & & 1 & & 1 & \end{array}$

satisfied. Coxeter and Conway associated a frieze pattern of order $N$ (i.e. with $N-1$ rows) to each triangulation of a regular polygon with $N$ sides and showed that every frieze pattern arises in this way. For an example see Figure 1.

In [5] and [16], the authors consider frieze patterns arising from Fomin-Zelevinsky cluster algebras of type $A_{N}[11,12]$. Motivated by the description [10] of cluster algebras of type $D_{N}$ in terms of (tagged) triangulations of a disc $S_{\odot}$ with a single puncture and $N$ marked points on the boundary we associate a (type D) frieze pattern to every such triangulation. Note that the type $D_{N}$ case of [10] has been described in detail by Schiffler [18] in his study of the corresponding cluster category.

Each number in our frieze pattern is the cardinality of a set of matchings of a certain kind between vertices of the triangulation and triangles in it, so our result can be regarded as a type $D$ version of a result of [7] (see [16]) giving the numbers in a Conway-Coxeter frieze pattern in terms of numbers of perfect matchings for graphs associated to the corresponding triangulation of an unpunctured disc.

We show further that, in the cases where the triangulation has a particularly nice form (corresponding to an orientation of the Dynkin diagram of type $D_{N}$ ), the numbers in our frieze pattern can be interpreted as specialisations of cluster variables of the cluster algebra of type $D_{N}$, c.f. similar results in type $A_{N}$ obtained by Propp [16] (for arbitrary triangulations). We note that this implies that in these cases our frieze patterns coincide with the frieze patterns of type $D_{N}$ described by P. Caldero in [4] based on [5], which form part of the motivation for this article. We remark that such frieze patterns were motivated by the corresponding cluster category (introduced for type $A$ in [6] and in general in [2]). In particular, the arrangement of the entries in the pattern corresponds to the Auslander-Reiten quiver of the cluster category. The frieze patterns we consider here are motivated in a similar way.

We remark that in independent work Musiker [15] has recently shown how to use perfect matchings to obtain explicit expressions for cluster variables in cluster algebras of type $A, B, C$ or $D$, in terms of an initial bipartite seed. Our work can be seen as complementary to this, since, while Musiker's approach works at the level of the cluster variables themselves, rather than their specialisations, it requires a specific choice of initial seed (corresponding to a particular tagged triangulation in the set-up here, up to symmetry).

We now go into more detail. Arcs in triangulations of $S_{\odot}$ can be indexed by ordered pairs $i, j$ of (possibly equal) boundary vertices $i, j$ together with unordered pairs $i, 0$ consisting of a vertex on the boundary together with the puncture, with the proviso that the pairs $i, i+1$ (where $i+1$ is interpreted as 1 if $i=N$ ) are not allowed. We denote the arcs by $D_{i j}$ and $D_{i 0}$ respectively. Let $m_{i j}$ and $m_{i 0}$ be positive integers associated to such arcs. We define a frieze pattern of type $D_{N}$ to be an array of numbers of the form in Figure 2 if $N$ is even or of the form in Figure 3 if $N$ is odd. The entries in the top row are all taken to be 1 . In the second row, the entries 


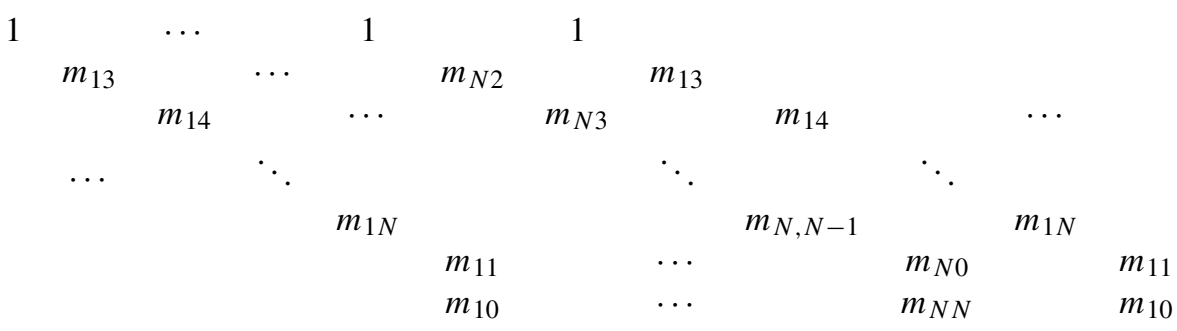

Fig. 2 A frieze pattern of type $D_{N}, N$ even

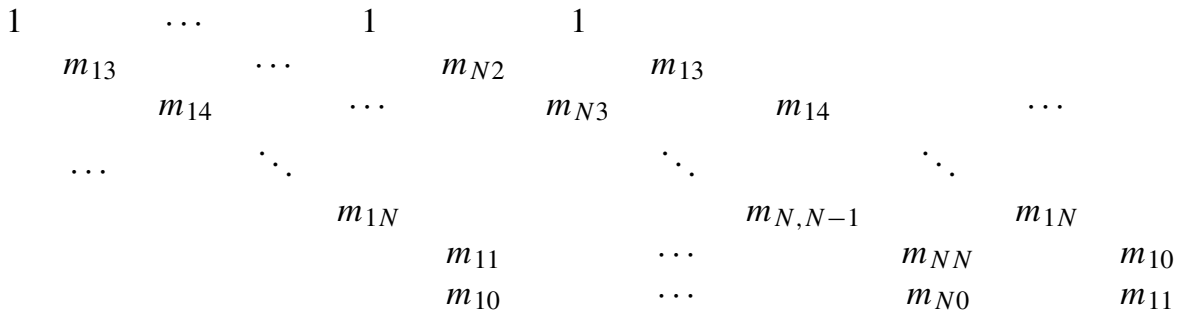

Fig. 3 A frieze pattern of type $D_{N}, N$ odd

are of the form $m_{i, i+2}$, corresponding to arcs connecting the vertex $i$ with the vertex $i+2$ (modulo $N$ ), arranged between the 1's of the top row. The third row gives the integers $m_{i, i+3}$ associated to arcs from $i$ to $i+3$ (modulo $N$ ), such that $m_{i, i+3}$ lies below the entries $m_{i, i+2}$ and $m_{i+1, i+3}$. The lowest two rows give the $m_{i, i}$ and $m_{i, 0}$, with the uppermost of the two rows giving the entries $m_{1,1}, m_{2,0}, m_{3,3}$ and so on, and the lowest row giving the entries $m_{1,0}, m_{2,2}, m_{3,0}$, and so on. Note that in the case where $N$ is odd, successive occurrences of the pair $m_{i 0}, m_{i i}$ above each other in the lowest two rows will be flipped (see Figure 4).

The following relations must be satisfied for all boundary vertices $i, j$ :

$$
\begin{aligned}
m_{i j} \cdot m_{i+1, j+1} & =m_{i+1, j} \cdot m_{i, j+1}+1, \text { provided } j \neq i \text { or } i+1 ; \\
m_{i, i-1} \cdot m_{i+1, i} & =m_{i+1, i-1} \cdot m_{i i} \cdot m_{i 0}+1 \\
m_{i i} \cdot m_{i+1,0} & =m_{i+1, i}+1 \\
m_{i 0} \cdot m_{i+1, i+1} & =m_{i+1, i}+1 .
\end{aligned}
$$

We refer to these relations as the frieze relations. For an example of a frieze pattern of type $D_{5}$ see Figure 4.

In Definition 2.14 we will explain how to associate numbers of matchings $m_{i j}$ and $m_{i 0}$ to a chosen triangulation of $S_{\odot}$ (with $N$ marked points on the boundary).

Theorem 1.1 If the matching numbers $m_{i j}$ are arranged as above, then they form a frieze pattern of type $D_{N}$. 


\begin{tabular}{|c|c|c|c|c|c|c|c|c|c|c|c|c|c|}
\hline & 1 & & 1 & & 1 & & 1 & & 1 & & & & \\
\hline$m_{13}$ & & $m_{24}$ & & $m_{35}$ & & $m_{41}$ & & $m_{52}$ & & $m_{13}$ & & & \\
\hline \multirow[t]{9}{*}{3} & & 3 & & 1 & & 2 & & 4 & & 3 & & & \\
\hline & $m_{14}$ & & $m_{25}$ & & $m_{31}$ & & $m_{42}$ & & $m_{53}$ & & $m_{14}$ & & \\
\hline & 8 & & 2 & & 1 & & 7 & & 11 & & 8 & & \\
\hline & & $m_{15}$ & & $m_{21}$ & & $m_{32}$ & & $m_{43}$ & & $m_{52}$ & & $m_{15}$ & \\
\hline & & 5 & & 1 & & 3 & & 19 & & 29 & & 5 & \\
\hline & & & $m_{11}$ & & $m_{20}$ & & $m_{33}$ & & $m_{40}$ & & $m_{55}$ & & $m_{10}$ \\
\hline & & & 2 & & 1 & & 4 & & 5 & & 6 & & 1 \\
\hline & & & $m_{10}$ & & $m_{22}$ & & $m_{30}$ & & $m_{44}$ & & $m_{50}$ & & $m_{11}$ \\
\hline & & & 1 & & 2 & & 2 & & 10 & & 3 & & 2 \\
\hline
\end{tabular}

Fig. 4 A frieze pattern of type $D_{5}$

Let $\mathcal{A}$ be a cluster algebra [11] of type $D_{N}$. We consider the case in which all coefficients are set to 1 . Let $x_{i j}$ be the cluster variable corresponding to the $\operatorname{arc}$ in $S_{\odot}$ with end-points $i$ and $j[10,18]$. Fix a seed $(\mathbf{x}, B)$ of $\mathcal{A}$, so that $\mathbf{x}$ is a cluster and $B$ is a skew-symmetric matrix. Let $Q$ be the quiver associated to $B$. Let $u_{i j}$ denote the integer obtained from $x_{i j}$ when the elements of $\mathbf{x}$ are all specialised to 1 .

Theorem 1.2 Suppose that the quiver $Q$ of the seed $(\mathbf{x}, B)$ is an orientation of the Dynkin diagram of type $D_{N}$. Let $D_{i j}$ be an arc in $S_{\odot}$. Then $m_{i j}=u_{i j}$.

In Section 2 we introduce the necessary notation and definitions, describing the matching numbers referred to above in detail. In Section 3 we give an example, and in Section 4 we recall the properties of the numbers in Conway-Coxeter frieze patterns that we need. In Section 5, we prove Theorem 1.1. In Section 6, we show how to associate frieze patterns to the more general tagged triangulations, and in Section 7 we prove Theorem 1.2 and make two conjectures. In an appendix to this article, Hugh Thomas proves one of these conjectures and a corrected version of the second.

\section{Notations and definitions}

We consider "triangulations" of bounded discs with a number of marked points on the boundary and up to one marked point in the interior (a puncture). Such a disc is a special case of a bordered surface with marked points as in recent work [10] of Fomin, Shapiro and Thurston - they consider surfaces with an arbitrary number of boundary components and punctures. We use the following notation: $S$ denotes a bounded disc with $N$ marked points (or boundary vertices) on the boundary, $N \geq 3$. We add a subscript $\odot$ if the disc has a puncture. We will usually label the points on the boundary clockwise around the boundary with $1,2, \ldots, N$ and denote the puncture by 0 .

For any such disc let $B_{i j}$ denote the boundary arc from the vertex $i$ up to the vertex $j$, going clockwise. So if $j=i+1, B_{i, i+1}$ denotes the boundary component from $i$ to $i+1$ including the two marked points. (Marked points are always taken $\bmod N$ ). If 
Fig. 5 The three types of triangles
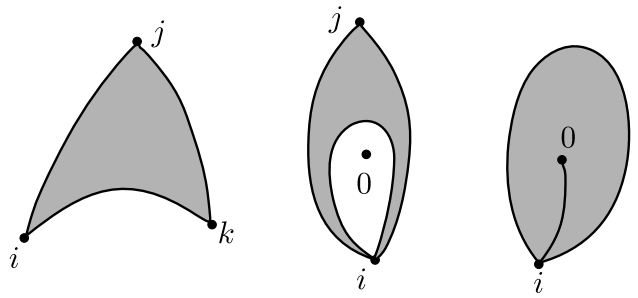

the disc has no puncture, we assume $i \neq j$. If it has a puncture then $i=j$ is allowed: $B_{i i}$ denotes the whole boundary, starting and ending at $i$.

Definition 2.1 An $\operatorname{arc} D$ of a disc is a curve whose endpoints are marked points of the disc and which does not intersect itself in the interior of the disc. Furthermore, we require that the interior of the arc is disjoint from the boundary of the disc and that it does not cut out an unpunctured monogon or digon. We consider arcs only up to isotopy.

We note that, in an unpunctured disc, given $i, j \in\{1, \ldots, N\}$ with $j \notin\{i-1, i, i+$ $1\}$, there is a unique $\operatorname{arc} D_{i j}=D_{j i}$ (up to isotopy) with endpoints $i, j$. In a punctured disc, given $i \in\{1, \ldots, N\}$, there are unique arcs $D_{i 0}$ (with endpoints $i$ and 0 ), $D_{i i}$ (with both endpoints $i$ ) and $D_{i, i-1}$ (with endpoints $i-1$ and $i$ ). For $i, j \in\{1, \ldots, N\}$ satisfying $j \notin\{i-1, i, i+1\}$, there are exactly two arcs, up to isotopy, with endpoints $i$ and $j$ : the arc $D_{i j}$ which winds partly around 0 , going clockwise from $i$ to $j$, and the $\operatorname{arc} D_{j i}$ which goes clockwise from $j$ to $i$, i.e. anticlockwise from $i$ to $j$. Note that for such $i, j$ we have $D_{i j}=D_{j i}$ if and only if the disc has no puncture. Note also that by definition, a boundary arc $B_{i, i+1}$ is not considered to be an arc.

Using the arcs, the discs can be triangulated:

Definition 2.2 A triangulation $\mathcal{T}$ of a disc $S$ (or $S_{\odot}$ ) is a maximal collection $\mathcal{T}$ of pairwise non-intersecting arcs of $S$ (of $S_{\odot}$ ).

By this we mean that we choose the representatives from the isotopy classes of arcs in such a way as to minimize intersections (and only choose one representative from any given isotopy class). It should be noted that arcs are allowed to intersect at the endpoints, i.e. arcs can meet at marked points.

The arcs of the triangulation divide the disc into a collection of disjoint triangles. We will often call them the triangles of $\mathcal{T}$. There are three types of triangles appearing, as shown in Figure 5. If the disc has no puncture, the only triangles appearing have three vertices and three arcs as sides. If the disc has a puncture, there are two more types of triangles, one with sides $D_{i j}, D_{j i}$ and $D_{i i}(i, j \neq 0)$ and one with the corresponding sides obtained by setting $j=0$. The latter is called a self-folded triangle. These three types correspond to the triangles of label $A_{1}, A_{2}$ and $A_{4}$ of Burman, cf. [3] and are called ideal triangles in [10] - our notion of triangulation is thus an ideal triangulation in [10]. 
The number of arcs in a triangulation is an invariant of the disc. It is clear that any triangulation of $S$ has $N-3$ arcs and $N-2$ triangles whereas triangulations of punctured discs $S_{\odot}$ have $N$ arcs and $N$ triangles.

We often need to refer to a subset $V_{k l}$ of the vertices of the disc:

Definition 2.3 For $1 \leq k, l \leq N$ we write $V_{k l}:=V\left(B_{k, l}\right)$ to denote the vertices of the boundary arc $B_{k, l}$. In other words, we are referring to the vertices from $k$ to $l$, going clockwise. In case $k>l$, the set $V_{k l}$ consists of the vertices $k, k+$ $1, \ldots, N, 1,2, \ldots, l$.

We will also need to consider truncations $\left(S_{i j}\right)_{\odot}$ and $S_{i j}$ of the punctured disc $S_{\odot}$, defined as follows:

Definition 2.4 Let $j \neq i+1$. Together with $D_{i j}$, the boundary arc $B_{j i}$ forms a (smaller) punctured disc which we denote by $\left(S_{i j}\right)_{\odot}$. Its vertices are $V_{j, i}$. In particular, $j$ is the clockwise neighbour of $i$ in $\left(S_{i j}\right)_{\odot}$.

On the other hand, $D_{i j}$ and $B_{i j}$ form an unpunctured disc which we denote by $S_{i j}$. Its vertices are $V_{i, j}$, where $i$ is the clockwise neighbour of $j$ in $S_{i j}$.

Note that for $D_{i j}$ we choose a representative of its isotopy class of arcs in a way as to minimize intersections with the $\operatorname{arcs}$ of $\mathcal{T}$.

In $S_{i j}$ and $\left(S_{i j}\right)_{\odot}$, some of the triangles of $\mathcal{T}$ may be cut open into several different regions. If $D_{i j}$ is not an arc of the triangulation, then there are triangles of $\mathcal{T}$ which are crossed by $D_{i j}$. If $S_{i j}$ contains two connected components of such a triangle we say that $D_{i j}$ splits the triangle.

Remark 2.5 If $D_{i j}$ is an arc of the triangulation, it does not cut across any triangles. Hence no split triangles appear and the $\operatorname{arcs}$ of $\mathcal{T}$ induce triangulations of $\left(S_{i j}\right)_{\odot}$ and of $S_{i j}$.

Definition 2.6 Let $S_{\odot}$ be a punctured disc with triangulation $\mathcal{T}$.

We denote by $\left.\mathcal{T}\right|_{S_{i j}}$ the subdivision of $S_{i j}$ into triangles and regions obtained from restricting the triangulation $\mathcal{T}$ to $S_{i j}$.

In the same way, $\left.\mathcal{T}\right|_{\left(S_{i j}\right)_{\odot}}$ denotes the subdivision of $\left(S_{i j}\right)_{\odot}$ into triangles and regions obtained from restricting $\mathcal{T}$ to $\left(S_{i j}\right)_{\odot}$.

Figure 6 presents two examples, one where no triangles are split and one where $D_{i j}$ splits triangles. Note that by the observations above, $\left.\mathcal{T}\right|_{S_{i j}}$ and $\left.\mathcal{T}\right|_{\left(S_{i j}\right)_{\odot}}$ are triangulations of the corresponding discs if $D_{i j} \in \mathcal{T}$.

Remark 2.7 Consider the arc $D_{i j}$ (where $j \neq i+1$ ) in a punctured disc $S_{\odot}$ with triangulation $\mathcal{T}$. If there is a $k$ in $V_{j, i}$ such that $\mathcal{T}$ contains the central arc $D_{k 0}$, then $\left.\mathcal{T}\right|_{S_{i j}}$ does not contain any pair of split triangles.

Given a triangulation of any disc (punctured or unpunctured) we are interested in the ways to allocate triangles to a subset $I$ of the vertices. That is we are interested 

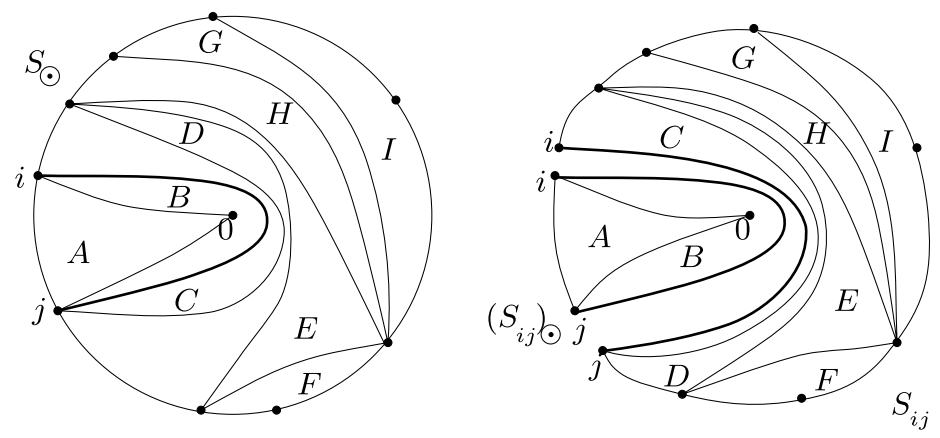

(a) no splitting
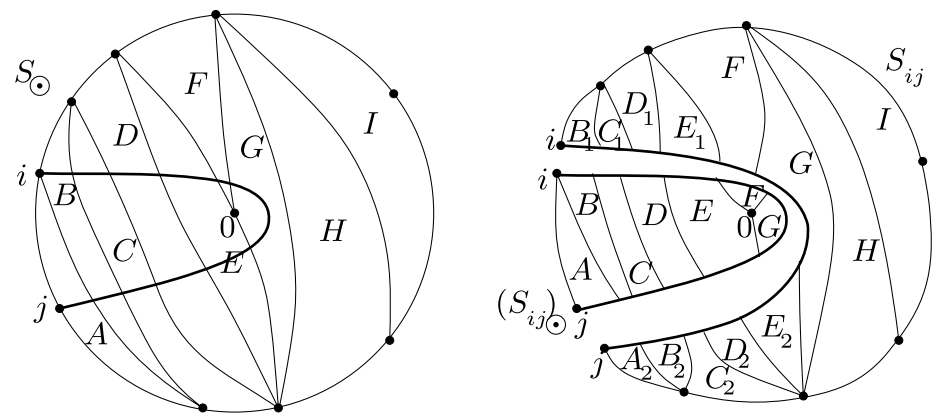

(b) splitting occurs

Fig. 6 Restricted triangulations through truncation by the arc $D_{i j}$

in matchings between a subset of the vertices and a subset of the triangles. In the case of a truncated version of a disc, we want to allocate triangles and regions of the truncated triangulation to vertices.

Definition 2.8 Let $S$ and $S_{\odot}$ be discs with vertices $\{1,2, \ldots, N\}, S_{\odot}$ with puncture 0 . Fix a triangulation $\mathcal{T}$ of the disc and let $I$ be a subset of the vertices $\{1, \ldots, N\}$ of cardinality $r$.

(i) A matching (for $S$ ) between $I$ and $\mathcal{T}$ is a way to allocate to each vertex $i \in I$ a triangle of $\mathcal{T}$ incident with $i$ in such a way that no triangle of $\mathcal{T}$ is allocated to more than one vertex.

(ii) A matching (for $S_{\odot}$ ) between $I \cup\{0\}$ and $\mathcal{T}$ is a way to allocate $r+1$ triangles of $\mathcal{T}$ to the vertices of $I$ and the puncture in the same way as above.

(iii) Given an arc $D_{i j}$ in $S_{\odot}$ let $S_{i j}$ be the truncated disc as in Definition 2.4 and let $I \subset V_{i, j}$ be a subset of the vertices between $i$ and $j$. Then a matching (for $S_{i j}$ ) between $I$ and $\left.\mathcal{T}\right|_{S_{i j}}$ is a way to allocate $r=|I|$ triangles or regions of $\left.\mathcal{T}\right|_{S_{i j}}$ to the vertices of $I$ as above. If a triangle is split the two regions appearing because of this are allowed to be allocated to different vertices. 

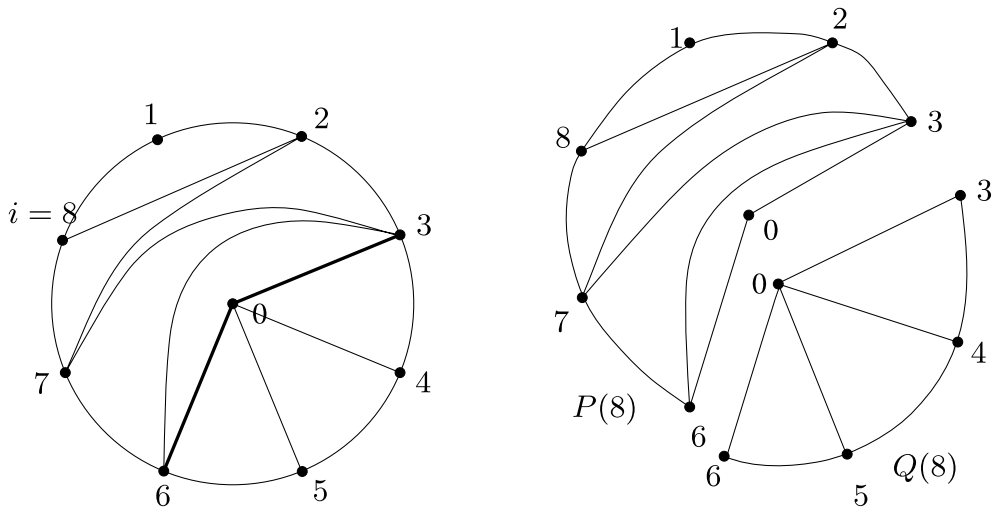

Fig. 7 Type (i), $i=8$
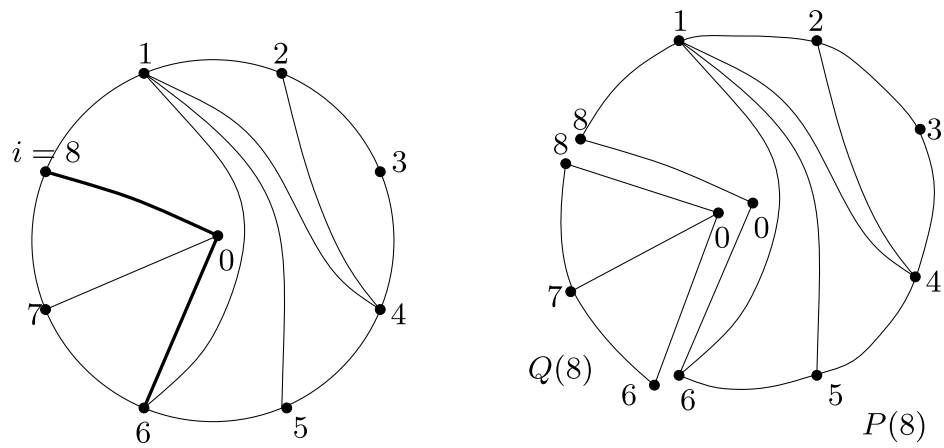

Fig. 8 Type (ii), $i=8$

Now if $\mathcal{T}$ is a triangulation of a disc (with or without puncture), we want to refer to the set of all matchings between a subset of the vertices of the disc and the triangles of $\mathcal{T}$ :

Definition 2.9 Let $\mathcal{T}$ be a triangulation of a disc and $I$ be a subset of the vertices $\{1, \ldots, N\}$ or of $\{1, \ldots, N\} \cup\{0\}$ in case the disc has a puncture. Then we set $\mathcal{M}(I, \mathcal{T})$ to be the set of all matchings between $I$ and $\mathcal{T}$.

Remark 2.10 Let $S_{\odot}$ be a punctured disc with $N$ marked points on the boundary and a fixed triangulation $\mathcal{T}$, and let $i \in\{1,2, \ldots, N\}$. Then we distinguish four cases. They are illustrated on the left hand sides of Figures 7, 8, 9 and 10:

(i) $\mathcal{T}$ contains at least two central arcs and $D_{i 0} \notin \mathcal{T}$;

(ii) $\mathcal{T}$ contains at least two central arcs and $D_{i 0} \in \mathcal{T}$;

(iii) There is exactly one central arc $D_{k 0}$ in the triangulation, $i \neq k$;

(iv) The only central $\operatorname{arc}$ of $\mathcal{T}$ is $D_{i 0}$. 

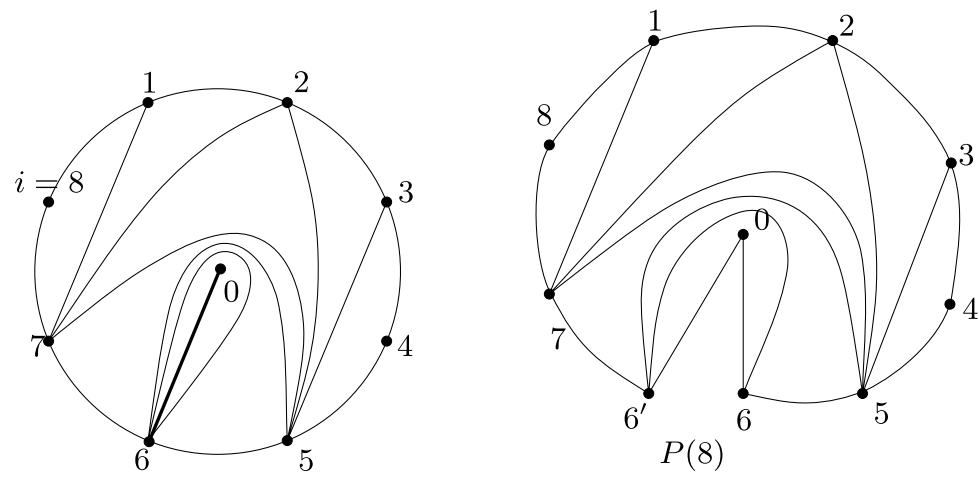

Fig. 9 Type (iii), $i=8$
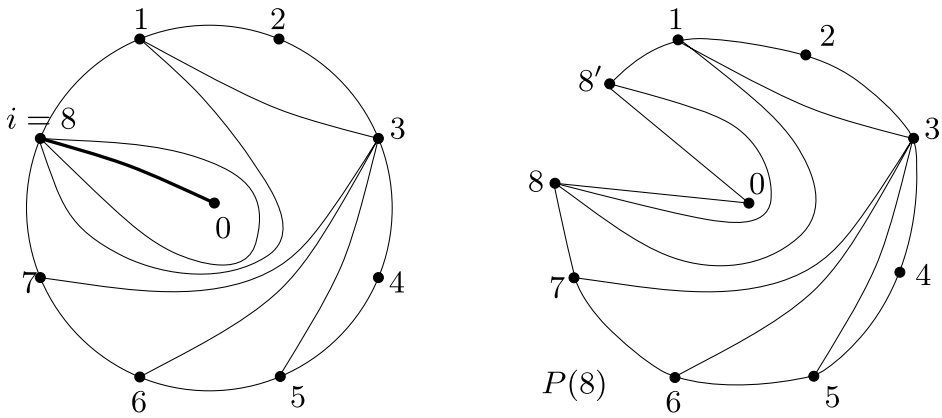

Fig. 10 Type (iv), $i=8$

We want to associate certain unpunctured $\operatorname{discs} P(i)$ and $Q(i)$ to the vertex $i$, in a similar way as we use an arc $D_{i j}$ to define the truncated discs $S_{i j}$ and $\left(S_{i j}\right)_{\odot}$ obtained from $S_{\odot}$. Here we use one or two central arcs to cut open $S_{\odot}$.

Definition 2.11 (i) Let $i$ be a marked point of $S_{\odot}$ and $j, k$ boundary vertices with $i \in V_{k j}$ such that $D_{j 0}, D_{k 0} \in \mathcal{T}$ and such that there is no $l \in V_{k j}$ other than $k, j$ with $D_{l 0} \in \mathcal{T}$. Then we let $P(i)$ be the unpunctured disc with boundary $B_{k j} \cup D_{j 0} \cup D_{k 0}$. On the other hand we let $Q(i)$ be the unpunctured disc with boundary $B_{j k} \cup D_{k 0} \cup$ $D_{j 0}$. Figure 7 illustrates this.

(ii) Let $i$ be a boundary vertex with $D_{i 0} \in \mathcal{T}$ and let $j$ be the nearest clockwise neighbour of $i$ with $D_{j 0} \in \mathcal{T}$. Then we let $P(i)$ be the unpunctured disc with boundary $B_{i j} \cup D_{j 0} \cup D_{i 0}$ and $Q(i)$ be the unpunctured disc with boundary $B_{j i} \cup D_{i 0} \cup D_{j 0}$. This is illustrated in Figure 8 .

(iii) If the only central arc of $\mathcal{T}$ is $D_{k 0}$ then we define $P(i)$ to be the unpunctured disc obtained by cutting up the disc at $k$, with boundary $B_{k k} \cup D_{k 0} \cup D_{k 0}$. We obtain an additional marked point on the boundary. We denote the anti-clockwise neighbour of 0 by $k$ and the clockwise neighbour of 0 by $k^{\prime}$. An example is presented in Figure 9 .

(iv) If the only central arc of $\mathcal{T}$ is $D_{i 0}$ then we define $P(i)$ to be the unpunctured disc obtained by cutting up the disc at $i$, with boundary $B_{i i} \cup D_{i 0} \cup D_{i 0}$. As before, 
we obtain an additional marked point on the boundary. We denote the anti-clockwise neighbour of 0 by $i$ and the clockwise neighbour of 0 by $i^{\prime}$. An example is presented in Figure 10.

In other words, in the first two cases we cut the disc $S_{\odot}$ along two central arcs and $Q(i)$ is the complement to $P(i)$. In the latter two cases we unfold the disc along the only central arc. In particular, $Q(i)$ is not defined in these cases.

Remark 2.12 The vertex $i$ and the puncture 0 are neighbours in $P(i)$ if and only if the arc $D_{i 0}$ is in the triangulation.

Definition 2.13 For $P(i)$ and $Q(i)$ as defined above, we let $\left.\mathcal{T}\right|_{P(i)}$ and $\left.\mathcal{T}\right|_{Q(i)}$ be the triangulations of $P(i)$ and $Q(i)$ obtained from $\mathcal{T}$ by only considering the triangles in $P(i)$ and in $Q(i)$ respectively. Then $\left.\mathcal{T}\right|_{P(i)}$ and $\left.\mathcal{T}\right|_{Q(i)}$ are triangulations of unpunctured discs.

With the notation introduced in Definition 2.9 we can now associate numbers $m_{i j}$ to a disc with a fixed triangulation.

Definition 2.14 Let $S_{\odot}$ be a punctured disc with vertices $\{1,2, \ldots, N\}$ and puncture 0 . Fix a triangulation $\mathcal{T}$ of $S_{\odot}$. Let $i, j \in\{1, \ldots, N\}, j \neq i, i+1$.

(i) Let $\mathcal{M}_{i j}:=\mathcal{M}\left(V_{i+1, j-1},\left.\mathcal{T}\right|_{S_{i j}}\right)$, i.e. the set of matchings between $V_{i+1, j-1}$ and $\left.\mathcal{T}\right|_{S_{i j}}$.

(ii) Let $\mathcal{M}_{i 0}:=\mathcal{M}\left(V(P(i)) \backslash\{i, 0\},\left.\mathcal{T}\right|_{P(i)}\right)$.

(iii) Let $\mathcal{M}_{i i}:=\mathcal{M}\left(V_{i+1, i-1} \cup\{0\}, \mathcal{T}\right)$.

We set $m_{i, i+1}=1, m_{i j}=\left|\mathcal{M}_{i j}\right|, m_{i 0}=\left|\mathcal{M}_{i 0}\right|$ and $m_{i i}=\left|\mathcal{M}_{i i}\right|$.

\section{An Example}

We consider an example of type $D_{8}$. We take the triangulation $\mathcal{T}$ of the punctured disc with 8 marked points on its boundary displayed in Figure 11. Each triangle has been labelled with a letter for reference. The corresponding frieze pattern is shown in Figure 12. Each entry in the frieze pattern (apart from the first row) is labelled by its corresponding diagonal.

For example, the entry under $D_{27}$ is 23 , since there are 23 matchings between $\left.\mathcal{T}\right|_{S_{27}}$ and the vertices $V_{3,6}=\{3,4,5,6\}$. Note that the triangles $D$ and $E$ are split by the arc $D_{27}$ and we include in the count matchings in which both resulting regions are allocated to vertices. Thus, for example, the matching $3 E 4 B 5 A 6 E$ is included.

The entry under $D_{52}$ is 5 , since there are 5 matchings between $\left.\mathcal{T}\right|_{S_{52}}$ and the vertices $V_{5,2}=\{6,7,8,1\}$. Note that in this case the arc $S_{52}$ does not split any triangles. The matchings are: $6 A 7 E 8 G 1 H, 6 D 7 E 8 G 1 H, 6 A 7 F 8 G 1 H, 6 D 7 F 8 G 1 H$, and $6 E 7 F 8 G 1 H$.

The entry under $D_{22}$ is 12 , since there are 12 matchings between $\mathcal{T}$ and the vertices $V_{31}=\{3,4,5,6,7,8,1\}$ and 0 . In this case each triangle can only be allocated to a single vertex, even triangle $F$ which is split by the arc $D_{22}$. The 12 matchings are shown in Figure 13. 
Fig. 11 A triangulation of a punctured octagon

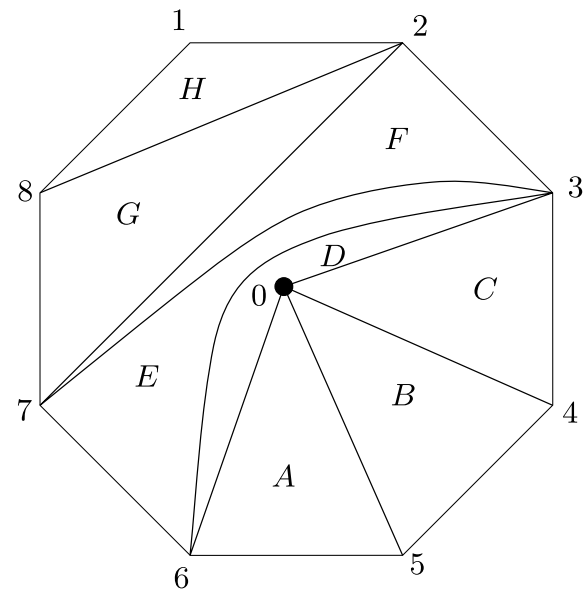

Fig. 12 A frieze pattern of type $D_{8}$

\section{The unpunctured case}

In this section we recall the frieze patterns of Conway and Coxeter and describe various interpretations of their entries and properties that they satisfy. We will phrase these results in terms of our set-up above.

In [8], [9], Conway and Coxeter studied frieze patterns of positive numbers. These are patterns of positive integers arranged in a finite number of rows where the top and bottom rows consist of 1's. The entries in the second row appear between the entries in the first row, the entries in the third row appear between the entries in the second

$$
b
$$

row, and so on. Also, for every diamond of the form $a \quad d$ the relation $a d-b c=1$

must be satisfied. The order $N$ of the pattern is one more than the number of rows. For an example see Figure 1.

Conway and Coxeter proved in $[8,9]$ that the second row of a frieze pattern of order $n$ is equal to the sequence of numbers of triangles at the vertices of a triangulation of a disc with $N$ marked points. Start with a vertex $i$ of $S$ and label it 0 . Whenever $i$ 
Fig. 13 Matchings for the arc $D_{22}$

$3 C 4 B 5 A 6 E 7 F 8 G 1 H 0 D$,
$3 D 4 B 5 A 6 E 7 F 8 G 1 H 0 C$,
$3 D 4 C 5 A 6 E 7 F 8 G 1 H 0 B$,
$3 D 4 C 5 B 6 E 7 F 8 G 1 H 0 A$,
$3 E 4 B 5 A 6 D 7 F 8 G 1 H 0 C$,
$3 E 4 C 5 A 6 D 7 F 8 G 1 H 0 B$,
$3 E 4 C 5 B 6 A 7 F 8 G 1 H 0 D$,
$3 E 4 C 5 B 6 D 7 F 8 G 1 H 0 A$,
$3 F 4 B 5 A 6 D 7 E 8 G 1 H 0 C$,
$3 F 4 C 5 A 6 D 7 E 8 G 1 H 0 B$,
$3 F 4 C 5 B 6 A 7 E 8 G 1 H 0 D$,
$3 F 4 C 5 B 6 D 7 E 8 G 1 H 0 A$.

$3 C 4 B 5 A 6 E 7 F 8 G 1 H 0 D$,

3D 4B 5A $6 E 7 F 8 G 1 H 0 C$,

$3 D 4 C 5 A 6 E 7 F 8 G 1 H 0 B$,

$3 D 4 C 5 B 6 E 7 F 8 G 1 H 0 A$,

(

$3 E 4 C 5 B 6 A 7 F 8 G 1 H 0 D$,

$3 E 4 C 5 B 6 D 7 F 8 G 1 H 0 A$,

$3 F 4 C 5 A 6 D 7 E 8 G 1 H 0 B$,

$3 F 4 C 5 B 6 A 7 E 8 G 1 H 0 D$,

$3 F 4 C 5 B 6 D 7 E 8 G 1 H 0 A$.

is connected to another vertex $j$ by an edge (including boundary edges) of $\mathcal{T}$, label $j$ by 1 . Furthermore, if $\Delta$ is a triangle with exactly two vertices which have been labelled already, label the third vertex with the sum of the other two labels. Iterating this procedure, we obtain labels for all vertices of $S$. The labels clearly depend on $i$ and the label obtained at $j$ (for $j \neq i$ ) is denoted by $(i, j)$.

Remark 4.1 Note that it is clear from the definition that $(i, i+1)=1$ for all $i$ and that $(i, j)=1$ whenever $i$ and $j$ are the two ends of an $\operatorname{arc}$ of $\mathcal{T}$.

The frieze pattern corresponding to $\mathcal{T}$ can then be described by displaying the numbers $(i, j)$ in the following way:

$$
\begin{aligned}
& (1,2) \\
& \begin{array}{lll}
\cdots & \cdots & (N-1, N) \\
& \cdots & (N-2, N)
\end{array} \\
& (1, N-1) \\
& (2, N)
\end{aligned}
$$

This fundamental region is then repeated upside down to the right and left, then the right way up on each side of that, and so on. Broline, Crowe and Isaacs have given the following interpretation of the numbers $(i, j)$.

Theorem 4.2 [1, Theorem 1] Let $S$ be a disc with $N$ marked points on the boundary with triangulation $\mathcal{T}$. Let $i, j$ be distinct marked points. Then:

(a) We have that $(i, j)$ is equal to $\left|\mathcal{M}\left(V_{i+1, j-1}, \mathcal{T}\right)\right|$, i.e. the number of matchings between $\mathcal{T}$ and $i+1, \ldots, j-1$.

(b) We have that $(i, j)=\left|\mathcal{M}\left(V_{j+1, i-1}, \mathcal{T}\right)\right|$.

In particular, $(i, j)=(j, i)$.

Definition 4.3 Let $i, j$ be a distinct pair of marked points on the boundary of $S$, and let $\mathcal{T}$ be a triangulation of $S$. Let $n_{i j}\left(=n_{i j}(\mathcal{T})\right)=|\mathcal{M}(\{1, \ldots, N\} \backslash\{i, j\}, \mathcal{T})|$, i.e. the number of matchings between $\{1,2, \ldots, N\} \backslash\{i, j\}$ and $\mathcal{T}$. 
Carroll and Price have shown that the numbers $(i, j)$ coincide with the $n_{i j}$ defined above. This is discussed in [16].

Theorem 4.4 [7] With the notation above, $(i, j)=n_{i j}$ for any pair of distinct vertices $i, j$.

We note the following corollary as we shall use it a lot later:

Corollary 4.5 With the notation above,

$$
n_{i j}=\left|\mathcal{M}\left(V_{i+1, j-1}, \mathcal{T}\right)\right|=\left|\mathcal{M}\left(V_{j+1, i-1}, \mathcal{T}\right)\right|,
$$

for any pair of distinct vertices $i, j$.

Propp reports that one of the main steps in the proof of Carroll and Price is the following, which is a direct consequence of the Condensation Lemma of Kuo [14, Theorem 2.5].

Proposition 4.6 Let $i, j, k, l$ be four boundary vertices of $S$ in clockwise order around the boundary of $S$. Then:

$$
n_{i k} n_{j l}=n_{i j} n_{k l}+n_{l i} n_{j k}
$$

Finally, we note that the numbers $(i, j)$ can be interpreted as specialisations of cluster variables by work of Fomin and Zelevinsky $[12,12.2]$. Let $\mathcal{A}$ be a cluster algebra of type $A_{N-3}$ with trivial coefficients. Then the cluster variables of $\mathcal{A}$ are in bijection with the set consisting of all of the diagonals of $S$ (using also [13]). For distinct vertices $i, j$, let $x_{i j}$ be the cluster variable corresponding to the diagonal $D_{i j}$ of $S$. By $[11,3.1], x_{i j}$ is a Laurent polynomial in the cluster variables corresponding to the diagonals of $\mathcal{T}$.

The following result is proved in $[16, \S 3]$ (for the $n_{i j}$ ), but we include a proof for the convenience of the reader. We also note that a connection between frieze patterns and cluster algebras was first explicitly given in [5].

Theorem 4.7 Let $i, j$ be distinct vertices on the boundary of $S$. Then $(i, j)$ is equal to the number $u_{i j}$ obtained from $x_{i j}$ by specialising each of the cluster variables corresponding to the arcs of $\mathcal{T}$ to 1 .

Proof This follows from [12] together with Theorem 4.4 and Proposition 4.6, which show that the $(i, j)$ and the $u_{i j}$ both satisfy the relations of Proposition 4.6. Since $(i, j)=1$ is equal to the specialisation of $x_{i j}$ whenever $D_{i j}$ is an $\operatorname{arc}$ in $\mathcal{T}$, the equality for arbitrary diagonals follows from iterated application of these relations.

\section{Construction of frieze patterns of type $D_{N}$}

Our aim in this section is to prove the main result, that the numbers $m_{i i}, m_{i j}$ and $m_{i 0}$ form a frieze pattern of type $D$. In order to do that we have to prove that the frieze 
relations (see Section 1) hold. We now work with the disc $S_{\odot}$ with puncture 0 and $N$ marked points on the boundary labelled $\{1,2, \ldots, N\}$. We first need the following result.

Lemma 5.1 Let $\mathcal{T}$ be a triangulation of $S_{\odot}$, and let $i, j \in\{1, \ldots, N\}$ with $j \neq i+1$.

(1) If $D_{i j} \in \mathcal{T}$ then $m_{i j}=1$.

(2) If $D_{i 0} \in \mathcal{T}$ then $m_{i 0}=1$.

Proof (1) Consider the truncated disc $S_{i j}$ with boundary $B_{i j}$ and $D_{i j}$. Since $D_{i j} \in \mathcal{T}$, none of the triangles of $\mathcal{T}$ are split by $D_{i j}$ and $\mathcal{T} \mid S_{i j}$ is a triangulation of $S_{i j}$. Suppose first that $i \neq j$. Then $m_{i j}$ is the number of matchings between $\{i+1, \ldots, j-1\}$ and $\left.\mathcal{T}\right|_{S_{i j}}$. Such a matching is a matching between $\left.\mathcal{T}\right|_{S_{i j}}$ and all of the vertices of the unpunctured disc $S_{i j}$ except $i$ and $j$ which are neighbours on the boundary of $S_{i j}$. By Remark 4.1, $m_{i j}=1$.

Suppose instead that $i=j$. Then $m_{i i}$ is the number of matchings between $\{1,2, \ldots, N\} \backslash\{i\} \cup\{0\}$ and $\mathcal{T}$. Such a matching is a matching between $\left.\mathcal{T}\right|_{S_{i i}}$ and all of the vertices of the unpunctured disc $P(i)$ except $i$ and $i^{\prime}$ which are joined by the $\operatorname{arc} D_{i i^{\prime}}$ in $P(i)$ induced by the $\operatorname{arc} D_{i i}$ of $\mathcal{T}$. By Remark 4.1, $m_{i i}=1$.

(2) If $D_{i 0} \in \mathcal{T}$ then $i$ and 0 are neighbours in $P(i)$ and the statement follows with the same reasoning.

Remark 5.2 We recall that in a triangulation of an unpunctured disc with $N$ marked points on the boundary, there are $N-2$ triangles. It follows that there are no matchings between a triangulation of the disc and a subset of the boundary vertices of cardinality greater than $N-2$.

Definition 5.3 Let $M$ be a matching between a subset $I$ of $\{1, \ldots, N\} \cup\{0\}$ and a triangulation $\mathcal{T}$ of $S_{\odot}$. Let $P$ be a subset of $S_{\odot}$ consisting of a union of triangles of $\mathcal{T}$. We denote by $\left.M\right|_{P}$ the restriction of $M$ to $P$ : this is the matching between $\left.\mathcal{T}\right|_{P}$ and $I \cap P$ obtained by allocating each vertex its corresponding triangle in $M$ whenever that triangle is contained in $P$.

In order to do some computations of numbers of matchings, we need the following simple observation:

Lemma 5.4 Let $\mathcal{T}$ be a triangulation of $S_{\odot}$, and let $S_{\odot}=P \cup Q$ be a decomposition of $S_{\odot}$ into two subsets with common boundary given by arcs of $\mathcal{T}$. Given a subset I of $\{1,2, \ldots, N\} \cup\{0\}$, let $J$ denote the subset of I consisting of vertices on the common boundary between $P$ and $Q$. Then the number of matchings between $\mathcal{T}$ and $I$ is given by

$$
|\mathcal{M}(I, \mathcal{T})|=\sum_{J^{\prime}, J^{\prime \prime}: J=J^{\prime} \sqcup J^{\prime \prime}} n_{J^{\prime}, J^{\prime \prime}}
$$

Here $n_{J^{\prime}, J^{\prime \prime}}$ is the number of matchings between $\mathcal{T}$ and $I$ in which all vertices of $J^{\prime}$ are allocated triangles in $P$ and all vertices of $J^{\prime \prime}$ are allocated triangles in $Q$. It is given by the product of the number of matchings between $\left.\mathcal{T}\right|_{P}$ and $P \cap\left(I \backslash J^{\prime \prime}\right)$ and the number of matchings between $\left.\mathcal{T}\right|_{Q}$ and $Q \cap\left(I \backslash J^{\prime}\right)$. 


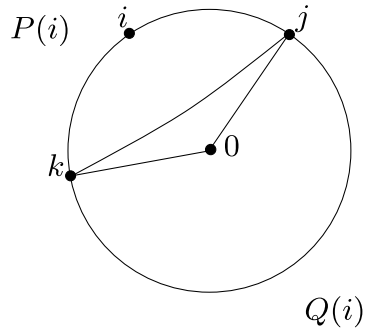

(a) Case I

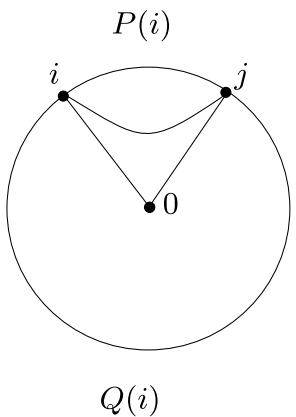

(b) Case II

Fig. 14 Proof of Lemma 5.5, Cases (I) and (II)

Proof Let $M$ be a matching between $I$ and $\mathcal{T}$. Given a pair $M_{P}, M_{Q}$ of matchings for $\left.\mathcal{T}\right|_{P}$ and $\left.\mathcal{T}\right|_{Q}$ which are compatible in the sense that any vertex of $J$ is allocated precisely one triangle in either $\left.\mathcal{T}\right|_{P}$ or $\left.\mathcal{T}\right|_{Q}$ (but not both), we can put them together to obtain a matching $M$ between $I$ and $\mathcal{T}$. This gives us a bijection between matchings $M$ between $I$ and $\mathcal{T}$ and compatible pairs $M^{\prime}, M^{\prime \prime}$ of matchings for $\left.\mathcal{T}\right|_{P}$ and $\left.\mathcal{T}\right|_{Q}$. The result follows from dividing up such pairs according to the allocation of the vertices of $J$ to triangles in $P$ or in $Q$.

Lemma 5.5 Let $\mathcal{T}$ be a triangulation of $S_{\odot}$. Let $d_{0}$ denote the number of triangles of $\mathcal{T}$ incident with the puncture, 0 , and let $i$ denote any boundary vertex of $S_{\odot}$. Then we have $d_{0} m_{i 0}=m_{i i}$.

Proof If there are at least two arcs incident with 0 in $\mathcal{T}$, let $j$ be the first boundary vertex strictly clockwise from $i$ such that $D_{j 0} \in \mathcal{T}$, and let $k$ be the first boundary vertex anticlockwise from $i$ (possibly equal to $i$ ) such that $D_{k 0} \in \mathcal{T}$. Since there are no arcs of the form $D_{l 0} \in \mathcal{T}$ for $l \in V_{k+1, j-1}$, we have $D_{k j} \in \mathcal{T}$. If there is only one arc $D_{l 0}$ incident with 0 in $\mathcal{T}$ then we take $j=k=l$. In this case $D_{j j} \in \mathcal{T}$.

Let $P(i)$ and $Q(i)$ be the unpunctured discs associated to $i$ in Definition 2.11 (where defined). For $a, b$ any distinct pair of boundary vertices of $P(i)$, let $p_{a b}$ denote the number of matchings between $\left.\mathcal{T}\right|_{P(i)}$ and all of the boundary vertices of $P(i)$ except $a$ and $b$ (thus $p_{a b}=n_{a b}\left(\left.\mathcal{T}\right|_{P(i)}\right)$; see Definition 4.3, noting that $P(i)$ is an unpunctured disc). Let $q_{a b}$ denote the corresponding number for a distinct pair of boundary vertices of $Q(i)$.

We consider four cases (I) - (IV), following the cases (i)-(iv) of Definition 2.11.

Case (I): We assume first that $i, j$ and $k$ are all distinct. See Figure 14(a). If, in a matching in $\mathcal{M}_{i i}$, both $j$ and $k$ are allocated triangles in $P(i)$ then 0 must be allocated a triangle in $Q(i)$ by Remark 5.2 applied to $P(i)$. By restriction of such a matching to $P(i)$ we obtain a matching for $\left.\mathcal{T}\right|_{P(i)}$ in which $i$ and 0 are not allocated triangles and by restriction to $Q(i)$ we obtain a matching for $\left.\mathcal{T}\right|_{Q(i)}$ in which $j$ and $k$ are not allocated triangles. Thus there are $p_{i 0} q_{j k}$ matchings in $\mathcal{M}_{i i}$ in which $j$ and $k$ are both allocated triangles in $P(i)$. Using the fact that $p_{i 0}=m_{i 0}$ (by definition) and Corollary 4.5 (which implies that $q_{j k}$ is the number of triangles at 0 in $Q(i)$, i.e., 
Fig. 15 Proof of Lemma 5.5, Case (III)
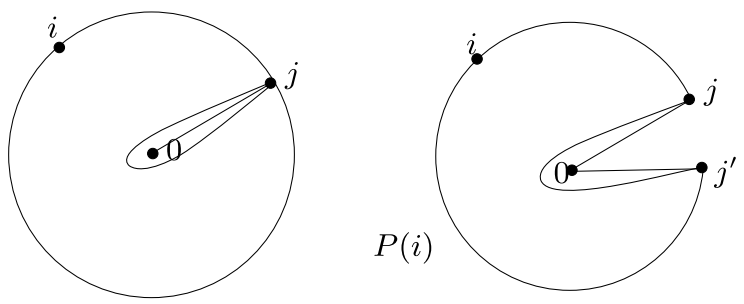

$\left.q_{j k}=d_{0}-1\right)$, we see that this is equal to $m_{i 0}\left(d_{0}-1\right)$. If $j$ is allocated a triangle in $Q(i)$ and $k$ is allocated a triangle in $P(i)$ then 0 is allocated a triangle in $P(i)$ by Remark 5.2 applied to $Q(i)$, and we obtain $p_{i j} q_{0 k}=p_{i j} \cdot 1=p_{i j}$ matchings of this type by Remark 4.1. Similarly, there are $p_{i k}$ matchings in $\mathcal{M}_{i i}$ in which $j$ is allocated a triangle in $P(i)$ and $k$ is allocated a triangle in $Q(i)$.

There are no matchings in $\mathcal{M}_{i i}$ in which both $j$ and $k$ are allocated triangles in $Q(i)$, by Remark 5.2 applied to $Q(i)$, so we have covered all cases. By Lemma 5.4 we have that $m_{i i}=\left|\mathcal{M}_{i i}\right|=\left(d_{0}-1\right) m_{i 0}+p_{i j}+p_{i k}$. By Proposition 4.6, $p_{i 0} p_{j k}=p_{i j} p_{0 k}+p_{i k} p_{0 j}$. Since $D_{k j} \in \mathcal{T}, p_{j k}=1$ by Remark 4.1, and we also have by Remark 4.1 that $p_{0 j}=p_{0 k}=1$, so $p_{i j}+p_{i k}=p_{i 0}=m_{i 0}$. We obtain

$$
m_{i i}=\left(d_{0}-1\right) m_{i 0}+m_{i 0}=d_{0} m_{i 0}
$$

as required.

Case (II): We assume next that $i=k$. See Figure 14. If, in a matching in $\mathcal{M}_{i i}, j$ is allocated a triangle in $P(i)$, then 0 must be allocated a triangle in $Q(i)$ by Remark 5.2 applied to $P(i)$, and we see that there are $p_{i 0} q_{i j}$ matchings of this type. By Corollary 4.5 this is equal to $m_{i 0}\left(d_{0}-1\right)=d_{0}-1$, noting that $m_{i 0}=1$ by Lemma 5.1. If $j$ is allocated a triangle in $Q(i)$, then 0 must be allocated a triangle in $P(i)$ by Remark 5.2 applied to $Q(i)$. We obtain $p_{i j} q_{0 i}=1 \cdot 1=1$ matchings of this type by Remark 4.1. By Lemma 5.4 we obtain a total number of matchings $m_{i i}=\left|\mathcal{M}_{i i}\right|=d_{0}-1+1=d_{0}=m_{i 0} d_{0}$ as required.

Case (III): We assume next that $i \neq j=k$. In a matching in $\mathcal{M}_{i i}$, only one of $j, j^{\prime}$ is allocated a triangle. See Figure 15.

We see that $m_{i i}$ is given by the sum of the number of matchings between $\left.\mathcal{T}\right|_{P(i)}$ and $V_{i+1, i-1} \cup\{0\}$ and the number of matchings between $\left.\mathcal{T}\right|_{P(i)}$ and $V_{i+1, i-1} \cup\{0\}$ with $j$ replaced by $j^{\prime}$. There are $p_{i j}$ matchings of the first kind and $p_{i j}$ matchings of the second kind, making a total of $p_{i j}+p_{i j^{\prime}}$. We have that $p_{i 0} p_{j j^{\prime}}=p_{i j} p_{0 j}+$ $p_{i j^{\prime}} p_{0 j^{\prime}}$, by Proposition 4.6, so $m_{i 0}=p_{i 0}=p_{i j}+p_{i j^{\prime}}$ by Remark 4.1, noting that $0, j$ and $0, j^{\prime}$ are adjacent on the boundary of $P(i)$ and $D_{j^{\prime} j}$ lies in $\left.\mathcal{T}\right|_{P(i)}$. We obtain $m_{i i}=m_{i 0}=m_{i 0} d_{0}$ as required.

Case (IV) We finally assume that $i=j=k$, so that there is a unique arc incident with 0 in $\mathcal{T}$ given by $D_{i 0}$. See Figure 16 . We have that $m_{i i}$ is given by the number of matchings between $V_{i+1, i-1} \cup\{0\}$ and $\left.\mathcal{T}\right|_{P(i)}$, so we obtain $m_{i i}=p_{i i^{\prime}}=1$ by Remark 4.1 since $\left.D_{i^{\prime} i} \in \mathcal{T}\right|_{P(i)}$. Hence $m_{i i}=d_{0} m_{i 0}$ as required, since $d_{0}=1$ and $m_{i 0}=1$ by Lemma 5.1 .

The proof of Lemma 5.5 is complete. 
Fig. 16 Proof of Lemma 5.5, Case (IV)
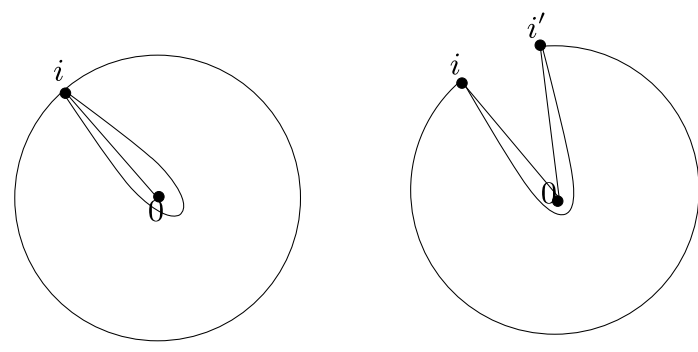

$P(i)$

As an example, we recall the type $D_{8}$-frieze pattern of Section 3: there, $m_{i i} / m_{i 0}=$ 4 (for all $i$ ), the number of triangles of $\mathcal{T}$ incident with 0 .

In order to prove the frieze relations hold, we first need the following:

Lemma 5.6 Let $\mathcal{T}$ be a triangulation of $S_{\odot}$. Let $i, j$ be boundary vertices of $S_{\odot}$ with $j \neq i+1$. Then the restriction $\left.\mathcal{T}\right|_{S_{i j}}$ can be extended to a triangulation of the entire disc $S$ with different marked points and the puncture removed.

Proof Let $a_{1}, a_{2}, \ldots, a_{t}$ be the vertices on the arc $B_{i, j}$ (distinct from $i$ and $j$ ) in order clockwise from $i$, which lie on arcs of $\mathcal{T}$ whose other end lies in $V_{j+1, i-1} \cup\{0\}$ For each $l$, let $b_{l, 1}, b_{l, 2}, \ldots, b_{l, u_{l}}$ be the end-points (other than $a_{l}$ ) of the $\operatorname{arcs}$ of $\mathcal{T}$ incident with $a_{l}$ whose other end lies in $V_{j+1, i-1} \cup\{0\}$.

The arc $D_{i, a_{1}}$ must lie in $\mathcal{T}$ if $a_{1} \neq i+1$, else the region on the $i$-side of the arc $D_{a_{1}, b_{l, 1}}$ will not be a triangle, since the other end-point of any arc incident with any of the vertices on the boundary arc $B_{i+1, a_{1}-1}$ can only be a vertex on the boundary arc $B_{i, a_{1}}$. Similarly, the arcs $D_{a_{m}, a_{m+1}}$ must all be in $\mathcal{T}$ if $a_{m+1} \neq a_{m}+1$, as must $D_{a_{t}, j}$ if $j \neq a_{t}+1$.

Remove the vertices $V_{j+1, i-1} \cup\{0\}$ and introduce new vertices on the boundary between $j$ and $i$ labelled

$$
c_{1,1}, c_{1,2}, \ldots, c_{1, u_{1}}, c_{2,1}, \ldots, c_{l, u_{l}},
$$

going anticlockwise from $i$ to $j$. We identify $c_{m, u_{m}}$ with $c_{m+1,1}$ for each $m$. We replace the part of each arc $D_{a_{m}, b_{l, m}}$ below the arc $D_{i, j}$ with a new arc linking $c_{l, m}$ with the intersection of $D_{a_{m}, b_{l, m}}$ and $D_{i, j}$, and add $\operatorname{arcs} D_{c_{1,1}, i}$ and $D_{j, c_{t, u_{t}}}$. See Figure 17 for an example. In this way we can complete $\left.\mathcal{T}\right|_{S_{i, j}}$ to a triangulation of the disc $S$, with new marked points on the boundary and the puncture removed, as required.

We first note the following consequence:

Lemma 5.7 Let $\mathcal{T}$ be a triangulation of $S_{\odot}$. Let $i, j$ be boundary vertices of $S_{\odot}$ with $j \neq i, i+1$. Then the numbers $m_{i i}, m_{i j}$ and $m_{i 0}$ as in Definition 2.14 are all positive.

Proof By Definition 2.14, the $m_{i 0}$ can be interpreted as entries in Conway-Coxeter frieze patterns (using Theorem 4.4), so are positive. By Lemma 5.6 the same argument applies to the $m_{i j}$. Then the $m_{i i}$ are positive by Lemma 5.5 . 
Fig. 17 Completing to a triangulation of the unpunctured disc

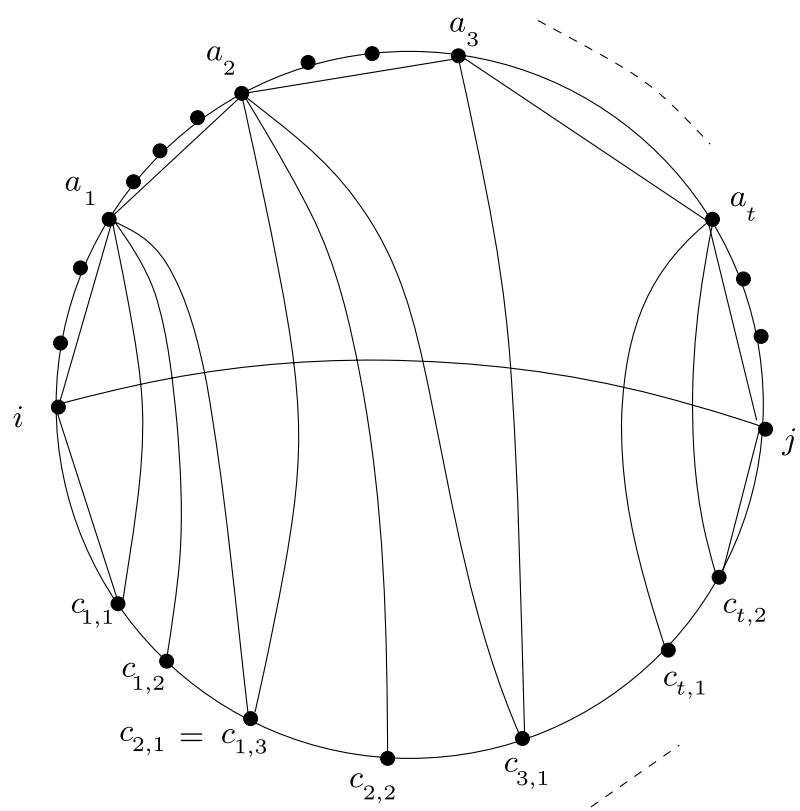

We can now prove that the frieze relations hold:

Proposition 5.8 Let $\mathcal{T}$ be a triangulation of $S_{\odot}$. Let $i, j$ be boundary vertices of $S_{\odot}$ with $j \neq i, i+1$. Let the numbers $m_{i i}, m_{i j}$ and $m_{i 0}$ be as in Definition 2.14. Then the following hold:

(1) $m_{i j} \cdot m_{i+1, j+1}=m_{i+1, j} \cdot m_{i, j+1}+1$

(2) $m_{i, i-1} \cdot m_{i+1, i}=m_{i+1, i-1} \cdot m_{i i} \cdot m_{i 0}+1$

(3) $\quad m_{i i} \cdot m_{i+1,0}=m_{i+1, i}+1$

(4) $m_{i 0} \cdot m_{i+1, i+1}=m_{i+1, i}+1$

i.e. the frieze relations (1.1) to (1.4).

Proof We prove each relation in turn.

Proof of (1): By Lemma 5.6 we can complete $\left.\mathcal{T}\right|_{S_{i, j+1}}$ to a triangulation $\mathcal{T}^{\prime}$ of the disc $S$ (with the puncture removed) and new marked points on the boundary. We see that $m_{i, j+1}$ is the number of matchings between $\mathcal{T}^{\prime}$ and $V_{i+1, j-1}$. It is clear that $m_{i j}$, $m_{i+1, j}$ and $m_{i, j+1}$ are the numbers of matchings between $\mathcal{T}^{\prime}$ and $V_{i+1, j-1}, V_{i+2, j-1}$ and $V_{i+1, j}$ respectively. Hence the frieze relation (1) holds by Proposition 4.6.

Proof of (2): We adopt the same notation for $j$ and $k$ as in the proof of Lemma 5.5.

Let $\widetilde{\mathcal{M}}_{i i}$ denote the set of matchings between $I:=\{1,2, \ldots, N\} \backslash\{i\}$ and $\left.\mathcal{T}\right|_{S_{i i}}$. Set $\widetilde{m}_{i i}=\left|\widetilde{\mathcal{M}}_{i i}\right|$. It is straightforward to show (along the lines of the proof of (1)) that, for any boundary vertex $i$ of $S_{\odot}$,

$$
m_{i, i-1} m_{i+1, i}=m_{i+1, i-1} \tilde{m}_{i i}+1 .
$$




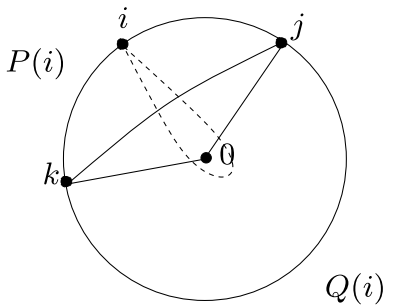

(a) Case I

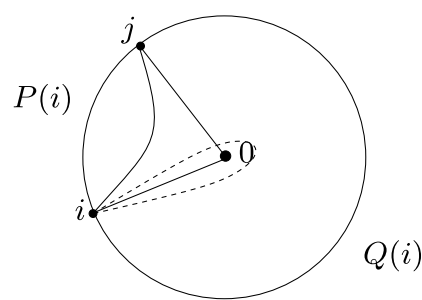

(b) Case II

Fig. 18 Proof of Proposition 5.8(2), Cases (I) and (II)

Hence for (2) it is enough to show that $\tilde{m}_{i i}=m_{i 0} m_{i i}$. By Lemma 5.5, this is equivalent to showing that $\tilde{m}_{i i}=d_{0} m_{i 0}^{2}$.

If there is more than one arc incident with 0 in $\mathcal{T}$, then we have a decomposition of the disc $S_{\odot}$ into smaller discs $P(i)$ and $Q(i)$ (see Definition 2.11). We shall apply Lemma 5.4 in these cases. Let $p_{a b}$ and $q_{a b}$ be as in the proof of Lemma 5.5. Again, we distinguish four cases (I)-(IV) to follow the cases (i)-(iv) of Definition 2.11.

Proof of (2), Case (I): We assume first that $i, j$ and $k$ are distinct. See Figure 18(a).

(a) Suppose first that in a matching in $\widetilde{\mathcal{M}}_{i i}, j, k$ are both allocated triangles in $P(i)$. Then restricting the matching to $P(i)$ we obtain a matching between $\left.\mathcal{T}\right|_{P(i)}$ and $V_{i+1, j} \cup V_{k, i-1}$. Since the arc $D_{i i}$ divides $P(i)$ into two parts, there are $\left|\mathcal{M}\left(V_{i+1, j},\left.\mathcal{T}\right|_{P(i)}\right)\right| \cdot\left|\mathcal{M}\left(V_{k, i-1},\left.\mathcal{T}\right|_{P(i)}\right)\right|$ of these. By Corollary 4.5 , this is equal to $m_{i 0}^{2}$. Restricting $\mathcal{T}$ to $Q(i)$, no triangles are split by $D_{i i}$. Therefore there are $\left|\mathcal{M}\left(V_{j+1, k-1},\left.\mathcal{T}\right|_{Q(i)}\right)\right|$ matchings of this type in $\widetilde{\mathcal{M}}_{i i}$. By Corollary 4.5 , this is equal to $\left|\mathcal{M}\left(\{0\},\left.\mathcal{T}\right|_{Q(i)}\right)\right|=d_{0}-1$. We see that there are $\left(d_{0}-1\right) m_{i 0}^{2}$ matchings in $\widetilde{\mathcal{M}}_{i i}$ in which $j$ and $k$ are both allocated triangles in $P(i)$.

(b) Arguing as above we see that there are $\left|\mathcal{M}\left(V_{k+1, i-1},\left.\mathcal{T}\right|_{P(i)}\right)\right| \cdot \mid \mathcal{M}\left(V_{i+1, j}\right.$, $\left.\left.\mathcal{T}\right|_{P(i)}\right) \mid$ possible restrictions to $P(i)$ of a matching in $\widetilde{\mathcal{M}}_{i i}$ in which $j$ is allocated a triangle in $P(i)$ and $k$ a triangle in $Q(i)$. By Corollary 4.5 this is equal to $p_{k i} p_{i 0}=p_{k i} m_{i 0}$. Similarly we see that there is $\mid \mathcal{M}\left(V_{j+1, k}, \mathcal{T}|Q(i)|=q_{j 0}=1\right.$ possible restriction to $Q(i)$, the last equality using Remark 4.1. We get a total of $p_{k i} m_{i 0}$ matchings of this type in $\widetilde{\mathcal{M}}_{i i}$.

(c) Arguing as in (b) we see that there are $p_{j i} m_{i 0}$ matchings in $\widetilde{\mathcal{M}}_{i i}$ in which $j$ is allocated a triangle in $Q(i)$ and $k$ a triangle in $P(i)$.

(d) By Remark 5.2 for $Q(i), j$ and $k$ cannot both be allocated triangles in $Q(i)$ for any matching in $\widetilde{\mathcal{M}}_{i i}$.

By Lemma 5.4 we see that $\tilde{m}_{i i}=\left(d_{0}-1\right) m_{i 0}^{2}+\left(p_{k i}+p_{i j}\right) p_{i 0}$. But by Proposition 4.6, $p_{i 0}=p_{i j}+p_{k i}$, so $\tilde{m}_{i i}=d_{0} m_{i 0}^{2}$ as required.

Proof of (2), Case (II): We next assume that $i=k \neq j$. See Figure 18(b).

(a) There are $\left|\mathcal{M}\left(V_{i+1, j},\left.\mathcal{T}\right|_{P(i)}\right)\right|$ possible restrictions to $P(i)$ of a matching in $\widetilde{\mathcal{M}}_{i i}$ in which $j$ is allocated a vertex in $P(i)$. There are $\left|\mathcal{M}\left(V_{j+1, i-1},\left.\mathcal{T}\right|_{Q(i)}\right)\right|$ possible restrictions to $Q(i)$, giving a total of

$$
\left|\mathcal{M}\left(V_{i+1, j},\left.\mathcal{T}\right|_{P(i)}\right)\right| \cdot\left|\mathcal{M}\left(V_{j+1, i-1},\left.\mathcal{T}\right|_{Q(i)}\right)\right|=p_{i 0}\left|\mathcal{M}\left(\{0\},\left.\mathcal{T}\right|_{Q(i)}\right)\right|=\left(d_{0}-1\right)
$$

by Corollary 4.5 and the fact that $p_{i 0}=m_{i 0}=1$ (by Lemma 5.1). 
Fig. 19 Proof of Proposition 5.8(2), Case (III)
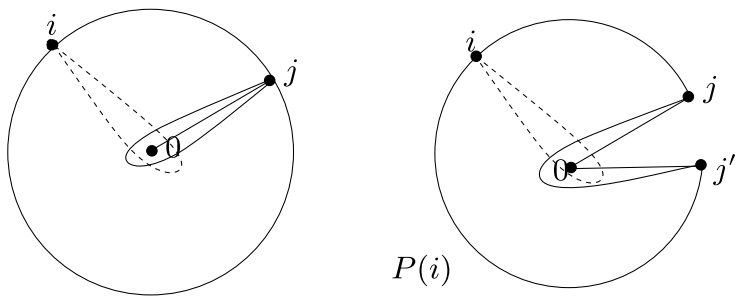

Fig. 20 Proof of Proposition 5.8(2), Case (IV)

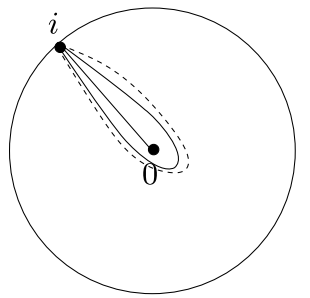

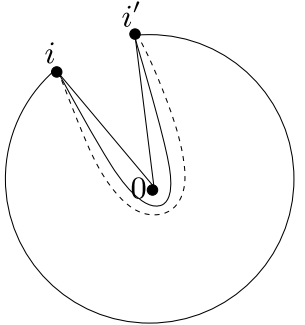

$P(i)$

(b) There are $\left|\mathcal{M}\left(V_{i+1, j-1},\left.\mathcal{T}\right|_{P(i)}\right)\right|$ possible restrictions to $P(i)$ of a matching in $\widetilde{\mathcal{M}}_{i i}$ in which $j$ is allocated a triangle in $Q(i)$. There are $\left|\mathcal{M}\left(V_{j, i-1},\left.\mathcal{T}\right|_{Q(i)}\right)\right|$ possible restrictions to $Q(i)$. By Corollary 4.5 the product of these is equal to $p_{i j} q_{i 0}$, which is equal to 1 by Lemma 5.1.

By Lemma 5.4, we have $\tilde{m}_{i i}=d_{0}-1+1=d_{0}$. This equals $m_{i 0}^{2} d_{0}$ by Lemma 5.1 so we are done.

Proof of (2), Case (III): We next assume that $j=k \neq i$. See Figure 19.

A matching in $\widetilde{\mathcal{M}}_{i i}$ induces a matching of $\left.\mathcal{T}\right|_{P(i)}$ in which either $j$ or $j^{\prime}$ is allocated a triangle but not both. Since $P(i)$ is split by $D_{i i}$ completely, there are $\left|\mathcal{M}\left(V_{j^{\prime}, i-1},\left.\mathcal{T}\right|_{P(i)}\right)\right| \cdot\left|\mathcal{M}\left(V_{i+1, j-1},\left.\mathcal{T}\right|_{P(i)}\right)\right|$ matchings of the first kind. By Corollary 4.5 this is equal to $p_{i 0} p_{i j}$. Similarly there are $p_{i j^{\prime}} p_{i 0}$ matchings of the second kind.

We get a total of $p_{i 0}\left(p_{i j}+p_{i j^{\prime}}\right)$ matchings in $\widetilde{\mathcal{M}}_{i i}$. By Proposition 4.6 we see that $p_{i 0}=p_{i j}+p_{i j^{\prime}}$, and we obtain $\tilde{m}_{i i}=p_{i 0}^{2}=m_{i 0}^{2}=d_{0} m_{i 0}^{2}$ as required, since $d_{0}=1$.

Proof of (2), Case (IV): Finally we consider the case where $i=j=k$. See Figure 20.

A matching in $\widetilde{\mathcal{M}}_{i i}$ induces a matching of $\left.\mathcal{T}\right|_{P(i)}$ in which all boundary vertices except $i$ and $i^{\prime}$ are allocated a triangle. Since $D_{i^{\prime} i}$ is an arc in $\left.\mathcal{T}\right|_{P(i)}$, we see by Remark 4.1 that there is only one possible such matching. So $\tilde{m}_{i i}=1=d_{0} m_{i 0}^{2}$, since $m_{i 0}=1$ by Lemma 5.1 .

Proof of (3): We note that by Lemma 5.5, it is sufficient to show that $d_{0} m_{i 0} m_{i+1,0}=$ $m_{i+1, i}+1$. Here, we distinguish six cases, labelled (Ia), (Ib), (IIa), (IIb), (III), (IV) where (Ia) and (Ib) correspond to (i) of Definition 2.11, etc.

Proof of (3), Case (Ia): We first assume that $i, i+1, j$ and $k$ are all distinct. See Figure 21(a).

(a) There are $\left|\mathcal{M}\left(V_{i+2, j},\left.\mathcal{T}\right|_{P(i)}\right)\right| \cdot\left|\mathcal{M}\left(V_{k, i-1},\left.\mathcal{T}\right|_{P(i)}\right)\right|$ possible restrictions to $P(i)$ of a matching in $\mathcal{M}_{i+1, i}$ in which both $j$ and $k$ are allocated triangles in $P(i)$, 


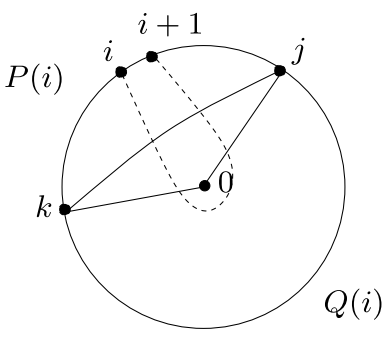

(a) Case Ia

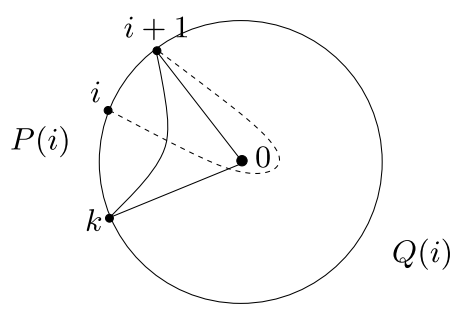

(b) Case Ib

Fig. 21 Proof of Proposition 5.8(3), Cases (Ia) and (Ib)

since $D_{i+1, i}$ splits $P(i)$ completely. By Corollary 4.5 (and the definition of $m_{i 0}$ and $\left.m_{i+1,0}\right)$, this equals $m_{i+1,0} m_{i 0}$. There are $\left|\mathcal{M}\left(V_{j+1, k-1},\left.\mathcal{T}\right|_{Q(i)}\right)\right|$ possible restrictions to $Q(i)$, which by Corollary 4.5 is equal to $\left|\mathcal{M}\left(\{0\},\left.\mathcal{T}\right|_{Q(i)}\right)\right|=d_{0}-1$. Hence there are $\left(d_{0}-1\right) m_{i 0} m_{i+1,0}$ matchings in $\mathcal{M}_{i+1, i}$ in total in which both $j$ and $k$ are allocated triangles in $P(i)$.

(b) There are $\left|\mathcal{M}\left(V_{i+2, j},\left.\mathcal{T}\right|_{P(i)}\right)\right| \cdot\left|\mathcal{M}\left(V_{k+1, i-1},\left.\mathcal{T}\right|_{P(i)}\right)\right|$ possible restrictions to $P(i)$ of a matching in $\mathcal{M}_{i+1, i}$ in which $j$ is allocated a triangle in $P(i)$ and $k$ is allocated a triangle in $Q(i)$. By Corollary 4.5 this is equal to $p_{i+1,0} p_{i k}=m_{i+1,0} p_{i k}$. There are $\left|\mathcal{M}\left(V_{j+1, k}, \mathcal{T} \mid Q(i)\right)\right|$ possible restrictions to $Q(i)$, which is equal to $q_{0 j}$ by Corollary 4.5 and thus equal to 1 by Remark 4.1. Thus we see that there are a total of $p_{i k} m_{i+1,0}$ matchings in $\mathcal{M}_{i+1, i}$ in which $j$ is allocated a triangle in $P(i)$ and $k$ is allocated a triangle in $Q(i)$.

(c) Arguing in a similar way to (b) we see that there are $p_{i+1, j} m_{i 0}$ matchings in $\mathcal{M}_{i+1, i}$ in which $j$ is allocated a triangle in $Q(i)$ and $k$ is allocated a triangle in $P(i)$.

(d) We note that it is not possible for both $j$ and $k$ to be allocated triangles in $Q(i)$ in a matching in $\mathcal{M}_{i+1, i}$, by Remark 5.2 applied to $Q(i)$.

By Lemma 5.4 we see that

$$
m_{i+1, i}=\left(d_{0}-1\right) m_{i 0} m_{i+1,0}+p_{i k} m_{i+1,0}+p_{i+1, j} m_{i 0} .
$$

By Proposition 4.6 we have that $p_{i 0} p_{j k}=p_{i j} p_{0 k}+p_{i k} p_{0 j}$, so $p_{i 0}=p_{i j}+p_{i k}$, noting that $D_{k j}$ is an $\operatorname{arc}$ in $\left.\mathcal{T}\right|_{P(i)}$, so $p_{j k}=1$. Hence

$$
\begin{aligned}
m_{i+1, i} & =\left(d_{0}-1\right) m_{i 0} m_{i+1,0}+m_{i+1,0}\left(p_{i 0}-p_{i j}\right)+m_{i 0} p_{i+1, j} \\
& =\left(d_{0}-1\right) m_{i 0} m_{i+1,0}+m_{i+1,0}\left(m_{i 0}-p_{i j}\right)+m_{i 0} p_{i+1, j} \\
& =\left(d_{0}-1\right) m_{i 0} m_{i+1,0}+m_{i 0} m_{i+1,0}-m_{i+1,0} p_{i j}+m_{i 0} p_{i+1, j} \\
& =\left(d_{0}-1\right) m_{i 0} m_{i+1,0}+m_{i 0} m_{i+1,0}-1 \\
& =d_{0} m_{i 0} m_{i+1,0}-1,
\end{aligned}
$$

using the fact that

$$
\begin{aligned}
p_{i+1,0} p_{i j} & =p_{i+1, j} p_{i 0}+p_{0 j} p_{i, i+1} \\
& =p_{i 0} p_{i+1, j}+1,
\end{aligned}
$$




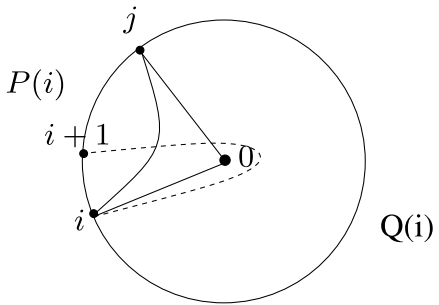

(a) Case IIa

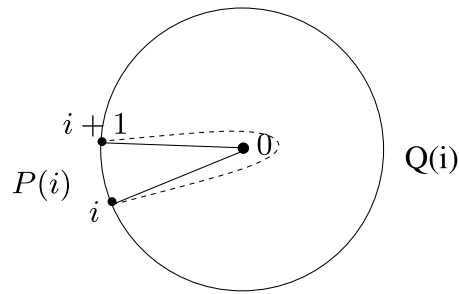

(b) Case IIb

Fig. 22 Proof of Proposition 5.8(3), Cases (IIa) and (IIb)

from Proposition 4.6. Hence $d_{0} m_{i 0} m_{i+1,0}=m_{i+1, i}+1$ as required.

Proof of (3), Case (Ib): We next assume that $i, i+1$ and $k$ are distinct while $i+1=j$. See Figure 21(b).

(a) There are $\left|\mathcal{M}\left(\{0\} \cup V_{k, i-1},\left.\mathcal{T}\right|_{P(i)}\right)\right|$ possible restrictions to $P(i)$ of a matching in $\mathcal{M}_{i+1, i}$ in which $k$ is allocated a triangle in $P(i)$. By Corollary 4.5 this is equal to $p_{i 0}=m_{i 0}$. There are $\left|\mathcal{M}\left(V_{i+2, k-1},\left.\mathcal{T}\right|_{Q(i)}\right)\right|$ possible restrictions to $Q(i)$, which by Corollary 4.5 is equal to $\left|\mathcal{M}\left(\{0\},\left.\mathcal{T}\right|_{Q(i)}\right)\right|=d_{0}-1$. We see that there are $\left(d_{0}-1\right) m_{i 0}$ matchings in $\mathcal{M}_{i+1, i}$ in which $k$ is allocated a triangle in $P(i)$.

(b) There are $\left|\mathcal{M}\left(V_{k+1, i-1},\left.\mathcal{T}\right|_{P(i)}\right)\right|$ possible restrictions to $P(i)$ of a matching in $\mathcal{M}_{i+1, i}$ in which $k$ is allocated a triangle in $Q(i)$. By Corollary 4.5 this is equal to $p_{k i}$. There are $\left|\mathcal{M}\left(V_{i+2, k},\left.\mathcal{T}\right|_{Q(i)}\right)\right|$ possible restrictions to $Q(i)$, which by Corollary 4.5 is equal to $q_{0, i+1}$. This equals 1 by Remark 4.1. We see that there are $p_{k i}$ matchings in $\mathcal{M}_{i+1, i}$ in which $k$ is allocated a triangle in $Q(i)$.

By Lemma 5.4 we obtain $m_{i+1, i}=\left(d_{0}-1\right) m_{i 0}+p_{k i}$. But by Proposition 4.6, we have that $p_{i 0}=p_{k i}+1$ (using the fact that $D_{i+1, k}$ is an arc in $\left.\mathcal{T}\right|_{P(i)}$ and Remark 4.1), so we get that $m_{i+1, i}=d_{0} m_{i 0}-1$, so $m_{i+1, i}+1=d_{0} m_{i 0} m_{i+1,0}$ as required, since $m_{i+1,0}=1$ by Lemma 5.1.

Proof of (3), Case (IIa): We next assume that $i, i+1$ and $j$ are distinct while $i=k$. See Figure 22(a).

It follows from symmetry with Case (Ib) above that $d_{0} m_{i+1,0}=m_{i+1, i}+1$, so $d_{0} m_{i+1,0} m_{i 0}=m_{i+1, i}+1$ as required, since $m_{i 0}=1$ by Lemma 5.1.

Proof of (3), Case (IIb): We next assume that $i=k$ and $i+1=j$. See Figure 22(b).

A matching in $\mathcal{M}_{i+1, i}$ is determined by its restriction to $Q(i)$. The number of possible restrictions is $\left|\mathcal{M}\left(V_{i+2, i-1},\left.\mathcal{T}\right|_{Q(i)}\right)\right|$ which is $\left|\mathcal{M}\left(\{0\},\left.\mathcal{T}\right|_{Q(i)}\right)\right|=d_{0}-1$ by Corollary 4.5. So $m_{i+1, i}=d_{0}-1$. Since $m_{i 0}=m_{i+1,0}=1$ by Lemma 5.1 we obtain $m_{i+1, i}+1=d_{0} m_{i 0} m_{i+1,0}$ as required.

Proof of (3), Case (III): We next assume that $i, i+1$ and $j$ are distinct, with $j=k$. See Figure 23.

There are $\left|\mathcal{M}\left(V_{i+2, j},\left.\mathcal{T}\right|_{P(i)}\right)\right| \cdot\left|\mathcal{M}\left(V_{j+1, i-1},\left.\mathcal{T}\right|_{P(i)}\right)\right|$ possible restrictions to $P(i)$ of a matching in $\mathcal{M}_{i+1, i}$ in which $j$ gets allocated a triangle, since $D_{i+1, i}$ splits $P(i)$ completely. There are $\left|\mathcal{M}\left(V_{i+2, j-1},\left.\mathcal{T}\right|_{P(i)}\right)\right| \cdot\left|\mathcal{M}\left(V_{j^{\prime}, i-1},\left.\mathcal{T}\right|_{P(i)}\right)\right|$ possible restrictions to $P(i)$ in which $j^{\prime}$ gets allocated a triangle. Thus the total number of matchings in $\mathcal{M}_{i+1, i}$ is the sum of these, which by Corollary 4.5 is equal to $p_{i+1,0} p_{j^{\prime} i}+p_{i+1, j} p_{0 i}$. By Proposition 4.6, $p_{i 0} p_{j j^{\prime}}=p_{i j} p_{0 j^{\prime}}+p_{i j^{\prime}} p_{0 j}$, so $p_{i 0}=$ 
Fig. 23 Proof of Proposition 5.8(3), Case (III)
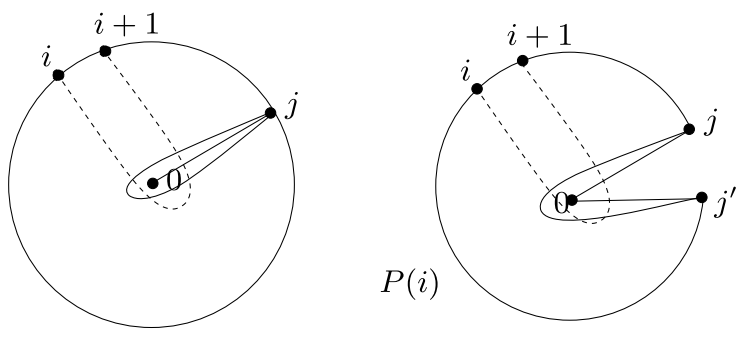

Fig. 24 Proof of Proposition 5.8(3), Case (IVa)

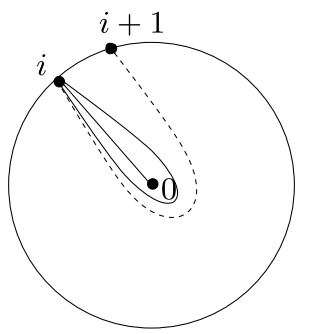

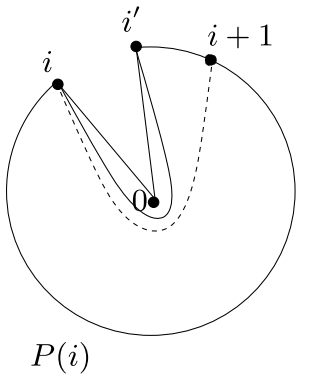

$p_{i j}+p_{i j^{\prime}}$. We thus have that

$$
\begin{aligned}
m_{i+1, i} & =m_{i+1,0}\left(p_{i 0}-p_{i j}\right)+m_{i 0} p_{i+1, j} \\
& =m_{i+1,0} m_{i 0}-m_{i+1,0} p_{i j}+m_{i 0} p_{i+1, j} \\
& =m_{i+1,0} m_{i 0}-1 \\
& =d_{0} m_{i 0} m_{i+1,0}-1,
\end{aligned}
$$

as required. Here we use the fact that

$$
p_{i+1,0} p_{i j}=p_{i+1, j} p_{i 0}+p_{0 j} p_{i, i+1}=p_{i 0} p_{i+1, j}+1
$$

from Corollary 4.5 and Remark 4.1, and the fact that $d_{0}=1$.

Proof of (3), Case (IVa): We assume that $i=j=k$. See Figure 24.

Since a matching in $\mathcal{M}_{i+1, i}$ is determined its restriction to $P(i)$, which is a matching between $\left.\mathcal{T}\right|_{P(i)}$ and $V_{i+2, i-1}$ we have that $m_{i+1, i}=\left|\mathcal{M}\left(V_{i+2, i-1},\left.\mathcal{T}\right|_{P(i)}\right)\right|$ which equals $p_{i+1, i}$ by Corollary 4.5. By Proposition 4.6, we have that

$$
p_{i+1,0} p_{i^{\prime} i}=p_{i+1, i} p_{0 i^{\prime}}+p_{i^{\prime}, i+1} p_{i 0},
$$

so, since $m_{i+1,0}=p_{i+1,0}$ (by definition of $m_{i+1,0}$ ), $m_{i+1, i}=p_{i+1, i}$ (by Corollary 4.5), and $p_{i^{\prime} i}=p_{0 i^{\prime}}=p_{i 0}=1$ (using Remark 4.1), we obtain $m_{i+1,0}=m_{i+1, i}+$ 1 , so $d_{0} m_{i 0} m_{i+1,0}=m_{i+1, i}+1$, as required, since $d_{0}=m_{i 0}=1$.

Proof of (3), Case (IVb): We assume that $i+1=j=k$. The argument for this case is analogous to that for Case (IVa).

Proof of (4): We note that $m_{i 0} m_{i+1, i+1}=m_{i 0} d_{0} m_{i+1,0}=m_{i i} m_{i+1,0}$ by Lemma 5.5, so (4) follows from (3). 
The proposition is proved.

Theorem 5.9 Let $\mathcal{T}$ be a triangulation of $S_{\odot}$. Then the numbers $m_{i j}, m_{i i}$ and $m_{i 0}$ in Definition 2.14 (arranged as in the introduction) form a frieze pattern of type $D_{N}$.

Proof This follows immediately from Proposition 5.8 and Lemma 5.7.

\section{Tagged triangulations}

In Section 5, we have established a way to obtain a frieze pattern of type $D_{N}$ from a triangulation of a punctured disc with $N$ boundary vertices using the matching numbers. Here, we will show that there exist frieze patterns (of type $D_{N}$ ) which cannot be obtained in the same way. We will describe another way to construct such frieze patterns and will show how they can be associated to tagged triangulations (as defined below).

Definition 6.1 We denote the frieze pattern of type $D_{N}$ associated in Theorem 5.9 to the triangulation $\mathcal{T}$ of a punctured disc by $F(\mathcal{T})$.

The frieze patterns of the form $F(\mathcal{T})$ do not give all possible frieze patterns of type $D_{N}$, as we will see now.

Definition 6.2 Let $P$ be a frieze pattern of type $D_{N}$. We define $\iota(P)$ to be the pattern obtained from $P$ by interchanging the last two rows.

Thus, if $\mathcal{T}$ is a triangulation of $S_{\odot}, \iota(F(\mathcal{T}))$ is also a frieze pattern of type $D_{N}$. If a triangulation has only one triangle at the puncture (i.e. $d_{0}=1$ ), then $m_{i i}=m_{i 0}$ for all $i$ (by Lemma 5.5), so the process of interchanging the last two rows does not give a new pattern, so $\iota(F(\mathcal{T}))=F(\mathcal{T})$. Otherwise, $\iota(F(\mathcal{T}))$ cannot be obtained via a triangulation of a punctured disc:

Remark 6.3 Let $\mathcal{T}$ be a triangulation of a punctured disc $S_{\odot}$. If $\mathcal{T}$ has at least two central arcs then there is no triangulation $\mathcal{T}^{\prime}$ of $S_{\odot}$ such that $\iota(F(\mathcal{T}))=F\left(\mathcal{T}^{\prime}\right)$.

Proof Let $F(\mathcal{T})$ be obtained from the matching numbers $m_{i j}$ of $\mathcal{T}$ and let $\iota(F(\mathcal{T}))$ be defined as above. Assume that $d_{0}>1$. Then since $m_{i 0}=d_{0} m_{i i}$ (Lemma 5.5) we have $m_{i 0}<m_{i i}$. Now if there exists a triangulation $\mathcal{T}^{\prime}$ of $S_{\odot}$ with matching numbers $m_{i j}^{\prime}$ giving rise to $\iota\left(F(\mathcal{T})\right.$ ), then by Lemma 5.5 (applied to $\mathcal{T}^{\prime}$ ), the matching numbers of $\mathcal{T}^{\prime}$ must satisfy $m_{i 0}^{\prime}<m_{i i}^{\prime}$ for all $i$. But by construction, $m_{i 0}^{\prime}=m_{i i}>m_{i 0}=m_{i i}^{\prime}$.

We thus have a way to construct a second frieze pattern for every triangulation with $d_{0}>1$. We now recall the definition of tagged arcs resp. of tagged edges as introduced in [10, Definition 7.1] for triangulated surfaces and then in [18, Section 2.1] for punctured polygons respectively. The idea is to attach labels to arcs in the punctured disc $S_{\odot}$ : 

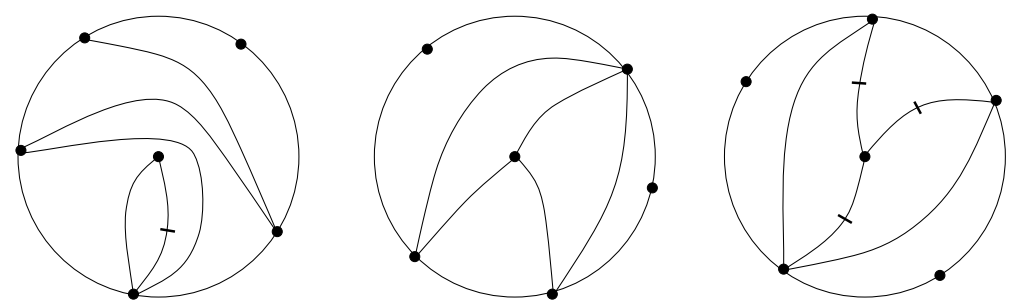

Fig. 25 Three tagged triangulations

Definition 6.4 Let $S_{\odot}$ be a punctured disc with $N$ boundary vertices with puncture 0 . The set of tagged arcs of $S_{\odot}$ is the following:

$$
\left\{D_{i j}^{1}, D_{i 0}^{1}, D_{i 0}^{-1} \mid 1 \leq i, j \leq N, j \neq i, i+1\right\} .
$$

We will often just write $D_{i j}$ instead of $D_{i j}^{1}$. When drawing tagged arcs, we indicate the label -1 of an arc by a short crossing line on it. Arcs labelled 1 are drawn as the usual arcs, cf. Figure 25.

Remark 6.5 Note that the tagged arcs are in bijection with the arcs in the sense of Definition 2.1: The arcs $D_{i j}^{1}(j \neq i, i+1)$ correspond to the usual arcs $D_{i j}(j \neq$ $i, i+1)$, the central $\operatorname{arcs} D_{i 0}^{1}$ to the usual central $\operatorname{arcs} D_{i 0}$ and the $\operatorname{arcs} D_{i 0}^{-1}$ of label -1 correspond to the loops $D_{i i}$.

Following [10, Definition 7.7] and [18, Section 2.4] we can now define tagged triangulations of punctured discs.

Definition 6.6 A tagged triangulation $\widetilde{\mathcal{T}}$ of $S_{\odot}$ is a collection of tagged arcs of $S_{\odot}$ obtained from a triangulation $\mathcal{T}$ of $S_{\odot}$ as follows:

(i) If $d_{0}=1$, replace the pair $D_{i i}, D_{i 0}$ by the tagged arcs $D_{i 0}^{-1}, D_{i 0}^{1}$ and replace the $\operatorname{arcs} D_{i j}$ by tagged $\operatorname{arcs} D_{i j}^{1}$.

(ii) If $d_{0}=k \geq 2$, replace all central $\operatorname{arcs} D_{i_{j}, 0}$ either by $k$ tagged $\operatorname{arcs} D_{i_{j}, 0}^{1}$ or by $k$ tagged $\operatorname{arcs} D_{i_{j}, 0}^{-1}$.

See Figure 25 for three tagged triangulations for a punctured disc with 5 boundary vertices.

In other words: if $\mathcal{T}$ is a triangulation of $S_{\odot}$ containing a unique central arc $D_{i 0}$ and hence also the loop $D_{i i}$ (in particular, $d_{0}=1$ ), then $\mathcal{T}$ gives rise to a tagged triangulation $\widetilde{\mathcal{T}}$ whose only central tagged arcs are $D_{i 0}^{1}$ and $D_{i 0}^{-1}$. If $\mathcal{T}$ has central arcs $D_{i_{1}, 0}, \ldots, D_{i_{k}, 0}, k \geq 2$ (so $d_{0}=k \geq 2$ ), then $\mathcal{T}$ gives rise to two tagged arc triangulations: one with $k$ central tagged arcs $D_{i_{j}, 0}^{1}$ and one with $k$ central tagged $\operatorname{arcs} D_{i_{j}, 0}^{-1}$.

Definition 6.7 Let $\widetilde{\mathcal{T}}$ be an arbitrary tagged triangulation of a punctured disc $S_{\odot}$. Then we associate a frieze pattern $F(\widetilde{\mathcal{T}})$ to it as follows: 
(a) If $\tilde{\mathcal{T}}$ has only arcs labelled 1 , let $\mathcal{T}$ be the triangulation of $S_{\odot}$ obtained via the bijection of Remark 6.5 . We then define $F(\widetilde{\mathcal{T}})$ as the frieze pattern of the matching numbers of $\mathcal{T}$, i.e. we set $F(\widetilde{\mathcal{T}})=F(\mathcal{T})$.

(b) If $\widetilde{\mathcal{T}}$ has exactly one tagged arc labelled -1 , say $D_{i 0}^{-1}$ and hence also $D_{i 0}^{1}$, then let $\mathcal{T}$ be the triangulation of $S_{\odot}$ obtained through the bijection in Remark 6.5. As in (a), we let $F(\widetilde{\mathcal{T}})$ be the frieze pattern $F(\mathcal{T})$ containing the matching numbers of $\mathcal{T}$.

(c) Suppose that $\widetilde{\mathcal{T}}$ has at least two arcs labelled -1 . Then every arc incident with 0 must be labelled -1 . Let $\mathcal{T}^{\prime}$ be the triangulation of $S_{\odot}$ consisting of all arcs $D_{i 0}$ such that $D_{i 0}^{-1}$ lies in $\widetilde{\mathcal{T}}$ and all arcs $D_{i j}$ (for $i$ and $j$ boundary vertices of $S_{\odot}$ ) such that $D_{i j}^{1}$ lies in $\widetilde{\mathcal{T}}$. Then we set $F(\widetilde{\mathcal{T}})=\iota\left(F\left(\mathcal{T}^{\prime}\right)\right)$.

We also note the following:

Proposition 6.8 The entries in a frieze pattern $M$ of type $D_{N}$ associated to a tagged triangulation $\tilde{\mathcal{T}}$ of a punctured disc are determined by the numbers of triangles incident with the marked points, together with the number of triangles incident with the puncture.

Proof Suppose first that $\mathcal{T}$ is a triangulation (without tags). We remark that it follows from the definition of $F(\mathcal{T})$ that an entry $m_{i, i+2}$ in the first row (with $1 \leq i \leq N$ ) is equal to the number of triangles incident with vertex $i$ in $\mathcal{T}$.

It is clear that, by induction on the rows, these entries determine the entries $m_{i j}$ in the pattern for all pairs of boundary vertices $i, j$ with $i \neq j$, via relation (1) in Proposition 5.8. Relations (2) and (3) can be used to determine $m_{i i} m_{i 0}$ for each boundary vertex $i$. Using Lemma 5.5 we see that all entries in $F(\mathcal{T})$ are determined in cases (a) and (b) of Definition 6.7. In case (c) we have $F(\widetilde{\mathcal{T}})=\iota(F(\mathcal{T}))$ and it is clear that the same approach works for such $\widetilde{\mathcal{T}}$.

\section{Cluster algebras and frieze patterns of type $D_{N}$}

Let $\mathcal{A}$ be a cluster algebra [11] of type $D_{N}$, as in the classification of cluster algebras of finite type [12]. We consider the case in which all coefficients are set to 1 . The algebra $\mathcal{A}$ is a subring of the rational function field $\mathbb{F}:=\mathbb{Q}\left(x_{1}, x_{2}, \ldots, x_{N}\right)$. It is determined by an initial seed, i.e. a pair consisting of a free generating set $\mathbf{x}_{0}$ of $\mathbb{F}$ over $\mathbb{Q}$ and a skew-symmetric integer matrix $B_{0}$ with rows and columns indexed by $\mathbf{x}_{0}$. For each element of $\mathbf{x}_{0}$, a new seed can be produced from $\left(\mathbf{x}_{0}, B_{0}\right)$ by a combinatorial process known as mutation. We obtain a collection of seeds via arbitrary iterative mutation of $\left(\mathbf{x}_{0}, B_{0}\right)$.

The free generating sets arising are known as clusters and $\mathcal{A}$ is generated by their union. The generators are known as cluster variables. We note that a skew-symmetric matrix $B$ appearing in a seed $(\mathbf{x}, B)$ can be encoded by a quiver, with vertices indexed by $\mathbf{x}$ and $b_{x y}$ arrows from the vertex indexed by $x$ to the vertex indexed by $y$ whenever $b_{x y}>0$.

By [10, Theorem 7.11] (see also [18]), the cluster variables of $\mathcal{A}$ are in bijection with the set of all tagged arcs in $S_{\odot}$, and the seeds of $\mathcal{A}$ are in bijection with the 
tagged triangulations of $S_{\odot}$. The matrix $B$ of a seed corresponding to a given tagged triangulation is described in [10, Definition 9.6].

Combining the above bijection between cluster variables and tagged arcs with the bijection in Remark 6.5, we obtain a bijection between cluster variables and the arcs of $S_{\odot}$ in the sense of Definition 2.1. For $D_{i j}$ (respectively, $D_{i 0}$ ) an arc of $S_{\odot}$, let $x_{i j}$ (respectively, $x_{i 0}$ ) denote the corresponding cluster variable.

Definition 7.1 Let $\widetilde{\mathcal{T}}$ be a tagged triangulation of $S_{\odot}$, and let $\mathbf{x}$ be the corresponding cluster of $\mathcal{A}$. We know from (a special case of) [11,3.1] that each cluster variable of $\mathcal{A}$ can be expressed as a Laurent polynomial in the elements of $\mathbf{x}$ with integer coefficients. For any arc $D_{i j}$ (respectively, $D_{i 0}$ ) of $S_{\odot}$, let $u_{i j}$ (respectively, $u_{i 0}$ ) denote the integer obtained from $x_{i j}$ (respectively, $x_{i 0}$ ) when the elements of $\mathbf{x}$ are all specialised to 1 .

Following $[4,5]$, let $F_{c}(\widetilde{\mathcal{T}})$ be the array of integers $u_{i j}$ written in the same positions as the $m_{i j}$ in Figures 2 and 3.

Proposition 7.2 For any tagged triangulation $\widetilde{\mathcal{T}}$, the array $F_{c}(\widetilde{\mathcal{T}})$ defined above is a frieze pattern of type $D_{N}$.

Proof (A similar proof in type $A$ is given in [5, §5]). If $D_{i j}$ (respectively, $D_{i 0}$ ) lies in $\mathcal{T}$, then $u_{i j}=1$ (respectively, $u_{i 0}=1$ ) is positive. The positivity of any entry in $F_{c}(\mathcal{T})$ then follows by induction on the number of mutations needed from $\mathbf{x}$ to obtain the corresponding cluster variable, using the exchange relations in $\mathcal{A}$. The frieze relations follow from some of the exchange relations in $\mathcal{A}$, i.e. those arising from [18, 5.1, 5.2] (using [5, 2.6(ii)]). The frieze relation (1.1) arises from case (1) of $[18,5.1]$ in the case where $a \neq d$ in Schiffler's notation. Relation (1.2) also arises from case (1), in the case where $a=d$. Relations (1.3) and (1.4) arise from case (2) of $[18,5.1]$. (Note that the exchange relations for a cluster algebra of type $D_{N}$ are also described in $[12,12.4])$.

Definition 7.3 A slice of a frieze pattern of type $D_{N}$ is defined as follows. We initially select an entry $E_{1}$ in the top row. For $2 \leq i \leq N-1$, suppose that an entry $E_{i-1}$ in the $i-1$ st row has already been chosen. We select an entry $E_{i}$ in the $i$ th row which is either immediately down and to the right of $E_{i-1}$ or immediately down and to the left of $E_{i-1}$. In addition, we select an entry $E_{N}$ in row $N$ below the entry immediately to the right of and below $E_{N-2}$ or below the entry immediately to the left of and below $E_{N-2}$.

Definition 7.3 is motivated by the notion of a slice in representation theory (see [17]).

Remark 7.4 Let $(\mathbf{x}, B)$ be a seed of $\mathcal{A}$. Let $\Gamma$ be the quiver associated to $B$ and let $\widetilde{\mathcal{T}}$ be a tagged triangulation. Then it can be checked using [10, Definition 9.6] that $\Gamma$ is an orientation of the Dynkin diagram of type $D_{N}$ if and only if the corresponding subset of $F_{c}(\widetilde{\mathcal{T}})$ is a slice in the above sense. 
Lemma 7.5 The entries in a slice of a frieze pattern of type $D_{N}$ determine the entire pattern.

Proof It is straightforward to see that the frieze relations can be used to determine all of the entries in the pattern.

Theorem 7.6 Suppose that the cluster $\mathbf{x}$ corresponds to a slice in the frieze pattern, and let $\widetilde{\mathcal{T}}$ be the corresponding tagged triangulation. Then the frieze patterns $F(\widetilde{\mathcal{T}})$ and $F_{c}(\widetilde{\mathcal{T}})$ coincide.

Proof As stated in Definition 6.7, $F(\widetilde{\mathcal{T}})$ is a frieze pattern of type $D_{N}$. By Proposition $7.2, F_{c}(\widetilde{\mathcal{T}})$ is a frieze pattern of type $D_{N}$. The entry in $F_{c}(\widetilde{\mathcal{T}})$ corresponding to any tagged arc $D$ of $\mathcal{T}$ is 1 by definition. If $\widetilde{\mathcal{T}}$ has at most one arc tagged with -1 then the entry of $F(\widetilde{\mathcal{T}})$ corresponding to $D$ is 1 by Lemma 5.1. If $\widetilde{\mathcal{T}}$ has more than one arc tagged with -1 then this entry is 1 by the definition of $F(\widetilde{\mathcal{T}})$ (Definition 6.7(c)).

The fact that $F(\widetilde{\mathcal{T}})$ and $F_{c}(\widetilde{\mathcal{T}})$ coincide then follows from iterative application of the relations of Proposition 5.8 and the frieze relations for $F_{c}(\widetilde{\mathcal{T}})$ (using Proposition 7.2). We use Lemma 7.5.

Finally, motivated by the situation for classical frieze patterns, we make the following conjectures:

Conjecture 7.7 Let $\widetilde{\mathcal{T}}$ be any tagged triangulation of $S_{\odot}$. Then $F(\widetilde{\mathcal{T}})$ and $F_{c}(\widetilde{\mathcal{T}})$ coincide.

Conjecture 7.8 Every frieze pattern of type $D_{N}$ is of the form $F(\widetilde{\mathcal{T}})$ for some tagged triangulation $\widetilde{\mathcal{T}}$ of $S_{\odot}$.

Acknowledgements The second author would like to thank Karin Baur and the ETH Zürich for their kind hospitality during a visit in autumn 2007. We would also like to thank the referees for their helpful comments on an earlier version of this paper.

\section{Appendix A: Resolution of Conjectures 7.7 and 7.8 (by Hugh Thomas)}

In this appendix, we prove Conjecture 7.7 of the paper. We show that Conjecture 7.8 is false as stated, but we prove a modified version of it.

Let $\mathcal{A}$ be the cluster algebra of type $D_{N}$ associated to the punctured disc $S_{\odot}$, over $k$ an arbitrary ground field of characteristic zero. $\mathcal{A}$ is, by definition, contained in some field of rational functions over $k$, so in particular, it is an integral domain. Write $K(\mathcal{A})$ for the field of fractions of $\mathcal{A}$.

Write $\Gamma$ for the set of (homotopy classes of) tagged arcs in $S_{\odot}$. Contained in $\mathcal{A}$ are the cluster variables $x_{\gamma}$ for $\gamma \in \Gamma$. The cluster variables, by definition, satisfy a collection of relations, called the exchange relations. These include the frieze relations (1.1)-(1.4) but there are more exchange relations than frieze relations. However, as we shall see below, in an important sense, the frieze relations suffice to define the cluster algebra. 
Let $X_{\Gamma}=\left\{X_{\gamma} \mid \gamma \in \Gamma\right\}$ be a set of indeterminates, and consider the polynomial ring $S=k\left[X_{\Gamma}\right]$ in this set of variables. Choose a triangulation $A$ of the punctured disc which corresponds to a slice of the frieze pattern. Write $S_{A}$ for the localization of $S$ at the indeterminates $X_{\alpha}$ with $\alpha \in A$.

Consider the map $\phi$ from $S_{A}$ to $K(\mathcal{A})$ which sends $X_{\gamma}$ to $x_{\gamma}$. Let $I$ be the kernel of this map. Since the elements $x_{\gamma}$ satisfy the frieze relations, $I$ contains an element expressing each of the frieze relations. (For example, corresponding to the frieze relation (1.1), we have elements of the form $X_{i j} X_{i+1, j+1}-X_{i+1, j} X_{i, j+1}-1$ in $I$.)

Lemma A.1 I is generated, as an ideal in $S_{A}$, by the frieze relations.

Proof Let $F$ denote the ideal of $S_{A}$ generated by the frieze relations. We write $X_{A}$ for the subset of $X_{\Gamma}$ corresponding to arcs of $A$, and similarly $x_{A}$ for the corresponding cluster of cluster variables.

Let $f \in I$. Suppose that some indeterminate $X_{\gamma}$ with $\gamma \notin A$ appears in $f$. By Lemma 7.5, the frieze relations determine an expression for $x_{\gamma}$ as a Laurent polynomial in terms of the elements $x_{A}$, say $x_{\gamma}=p_{\gamma}\left(x_{A}\right)$. Thus, since $g_{\gamma}=X_{\gamma}-p_{\gamma}\left(X_{A}\right)$ is an element of $S_{A}$, we have that $g_{\gamma} \in F$.

We can use this element to eliminate $X_{\gamma}$ from $f$. Applying the same argument repeatedly, we find that $f$ can be written as some $S$-linear combination of the elements $g_{\gamma}$ for $\gamma \notin A$, plus some remainder $\tilde{f}$ in which the only indeterminates that appear are elements of $X_{A}$. Since the elements $g_{\gamma}$ are in $F$ and therefore in $I$, it follows that $\tilde{f}$ must be in $I$, or in other words, $\phi(\tilde{f})=0$. The elements $x_{\alpha}$ are algebraically independent for $\alpha \in A$, so $\phi$ is injective on $k\left[X_{A}^{ \pm}\right]$. Thus $\tilde{f}$ must be zero. It follows that $f \in F$, and thus that $F=I$.

From the above lemma, it follows that $\phi$ determines an injection from $S_{A} / F$ to $K(\mathcal{A})$. Note that $\mathcal{A}$ is contained in the image of $\phi$. Thus $\phi$ allows us to identify $\mathcal{A}$ as a subring of $S_{A} / F$.

Let $\mathcal{F}$ be a frieze pattern of type $D$. Define $\hat{\psi}_{\mathcal{F}}: S \rightarrow k$ which sends $X_{\gamma}$ to the corresponding entry of the frieze pattern. Since the entries of the frieze pattern are by definition positive integers, and hence invertible in $k, \hat{\psi}_{\mathcal{F}}$ descends to $S_{A}$. Because the entries of the frieze pattern satisfy the frieze relations, $\hat{\psi}_{\mathcal{F}}$ descends further, to $S_{A} / F$, and thus determines a map $\psi_{\mathcal{F}}: \mathcal{A} \rightarrow k$.

We are now ready to prove Conjecture 7.7.

Proof of Conjecture 7.7 Let $\mathcal{T}$ be a triangulation. It determines two frieze patterns, $\mathcal{F}(\mathcal{T})$ and $\mathcal{F}_{c}(\mathcal{T})$, and thus two maps from $\mathcal{A}$ to $k$, namely $\psi_{\mathcal{F}(\mathcal{T})}$ and $\psi_{\mathcal{F}_{c}(\mathcal{T})}$.

As in the proof of Theorem 7.6, note that $\psi_{\mathcal{F}(\mathcal{T})}\left(x_{\gamma}\right)=1=\psi_{\mathcal{F}_{c}(\mathcal{T})}\left(x_{\gamma}\right)$, for $\gamma \in$ $\mathcal{T}$. By the Laurent phenomenon [11], any cluster variable in $\mathcal{A}$ can be expressed as a Laurent polynomial in the variables $x_{\mathcal{T}}$. Thus, $\psi_{\mathcal{F}(\mathcal{T})}$ and $\psi_{\mathcal{F}_{c}(\mathcal{T})}$ coincide. This implies that $\mathcal{F}(\mathcal{T})$ and $\mathcal{F}_{c}(\mathcal{T})$ also coincide. 
We now consider Conjecture 7.8. As stated, the conjecture is false. For example, consider the following frieze pattern of type $D_{4}$.

$\begin{array}{cccccc}1 & 1 & 1 & 1 & \\ 2 & 2 & 2 & 2 & \\ 3 & 3 & 3 & 3 & \\ 2 & 2 & 2 & 2 \\ 2 & 2 & 2 & 2\end{array}$

This frieze pattern does not arise as the frieze pattern of a tagged triangulation since such frieze patterns must have at least one 1 in the bottom two rows, because there is some edge connecting the puncture to a marked point on the boundary.

However, we can prove the following revision of the conjecture.

Proposition A.2 Any frieze pattern with at least one 1 in the bottom two rows corresponds to some tagged triangulation.

Proof Let $\mathcal{F}$ be a frieze pattern with at least one 1 in the bottom two rows. Without loss of generality, we can assume that there is an entry of the form $m_{i 0}$ which equals 1. This entry corresponds to an arc $\alpha$ connecting a boundary vertex to the puncture. The (untagged) arcs compatible with $\alpha$ are precisely the arcs of the unpunctured disc $P$ obtained by cutting open the punctured disc along $\alpha$.

The cluster variables corresponding to arcs of $P$ generate a subalgebra of $\mathcal{A}$, which we will denote $\mathcal{B}$. In a natural way, $\mathcal{B}$ is a cluster algebra of type $A_{N-1}$, in which $x_{i 0}$ appears as a coefficient. Now consider the entries in $\mathcal{F}$ which correspond to arcs of $P$. Since $\psi_{\mathcal{F}}\left(x_{i 0}\right)=1$, these entries satisfy the type $A_{N-1}$ exchange relations with no coefficients. Thus, in particular, if these numbers are rearranged into a type $A_{N-1}$ frieze pattern, they satisfy the frieze relations.

Now, by the type $A_{N-1}$ result of Coxeter and Conway [8,9], this type $A_{N-1}$ frieze pattern corresponds to a triangulation $\mathcal{T}^{\prime}$ of $P$, which, combined with $\alpha$, yields a triangulation $\mathcal{T}$ of the punctured disc. We know that $\psi_{\mathcal{F}(\mathcal{T})}\left(x_{\gamma}\right)=\psi_{\mathcal{F}}\left(x_{\gamma}\right)$ for $\gamma$ an arc of $P$ or $\gamma=\alpha$. In particular, $\psi_{\mathcal{F}(\mathcal{T})}$ and $\psi_{\mathcal{F}}$ agree for every $\gamma \in \mathcal{T}$. As in the proof of Conjecture 7.7 , it follows that $\psi_{\mathcal{F}}$ and $\psi_{\mathcal{F}(\mathcal{T})}$ coincide, and thus that $\mathcal{F}=\mathcal{F}(\mathcal{T})$, so $\mathcal{T}$ is the desired triangulation.

Acknowledgements The author was supported by an NSERC Discovery Grant. The appendix was written during a visit to NTNU; he thanks the Institutt for Matematiske Fag for its hospitality. He would also like to thank Bethany R. Marsh and Karin Baur for their comments on the original draft of this appendix.

\section{References}

1. Broline, D., Crowe, D.W., Isaacs, I.M.: The geometry of frieze patterns. Geom. Dedic. 3, 171-176 (1974)

2. Buan, A.B., Marsh, B.R., Reineke, M., Reiten, I., Todorov, G.: Tilting theory and cluster combinatorics. Adv. Math. 204, 572-618 (2006)

3. Burman, Y.M.: Triangulations of disc with boundary and the homotopy principle for functions without critical points. Ann. Glob. Anal. Geom. 17, 221-238 (1999) 
4. Caldero, P.: Algèbres cluster et catégories cluster, talks at the meeting on Algèbres Clusters, Luminy, France, May 2005

5. Caldero, P., Chapoton, F.: Cluster algebras as Hall algebras of quiver representations. Commun. Math. Helv. 81, 595-616 (2006)

6. Caldero, P., Chapoton, F., Schiffler, R.: Quivers with relations arising from clusters ( $A_{n}$ case). Trans. Am. Math. Soc. 358, 1347-1364 (2006)

7. Carroll, G., Price, G.: Two new combinatorial models for the Ptolemy recurrence. Unpublished memo (2003)

8. Conway, J.H., Coxeter, H.S.M.: Triangulated discs and frieze patterns. Math. Gaz. 57, 87-94 (1973)

9. Conway, J.H., Coxeter, H.S.M.: Triangulated discs and frieze patterns. Math. Gaz. 57, 175-186 (1973)

10. Fomin, S., Shapiro, D., Thurston, D.: Cluster algebras and triangulated discs. Part I: Cluster complexes. Preprint arxiv:math.RA/0608367v3 (2006)

11. Fomin, S., Zelevinsky, A.: Cluster algebras I: Foundations. J. Am. Math. Soc. 15(2), 497-529 (2002)

12. Fomin, S., Zelevinsky, A.: Cluster algebras II: Finite type classification. Invent. Math. 154(1), 63-121 (2003)

13. Fomin, S., Zelevinsky, A.: $Y$-systems and generalized associahedra. Ann. Math. 158(3), 977-1018 (2003)

14. Kuo, E.: Applications of graphical condensation for enumerating matchings and tilings. Theor. Comput. Sci. 319, 29-57 (2004)

15. Musiker, G.: A graph theoretic expansion formula for cluster algebras of type $B_{n}$ and $D_{n}$. Preprint arXiv:0710.3574v1 [math.CO] (2007)

16. Propp, J.: The combinatorics of frieze patterns and Markoff numbers. Preprint arXiv:math.CO/ 0511633 (2005)

17. Ringel, C.M.: Tame Algebras and Integral Quadratic Forms. Lecture Notes in Mathematics, vol. 1099. Springer, Berlin (1984)

18. Schiffler, R.: A geometric model for cluster categories of type $D_{n}$. Preprint arXiv:math.RT/0608264 (2006) 